DAMTP 98-88

hep-th/9807186

\title{
Superconformal Symmetry in Six-dimensions and Its Reduction to Four
}

\author{
Jeong-Hyuck Park* \\ Department of Applied Mathematics and Theoretical Physics \\ University of Cambridge \\ Silver Street, Cambridge, CB3 9EW, England
}

\begin{abstract}
Superconformal symmetry in six-dimensions is analyzed in terms of coordinate transformations on superspace. A superconformal Killing equation is derived and its solutions are identified in terms of supertranslations, dilations, Lorentz transformations, $R$-symmetry transformations and special superconformal transformations. The full superconformal symmetry, which is shown to form the group $\operatorname{OSp}(2,6 \mid N)$, is possible only if the supersymmetry algebra has $N$ spinorial generators of the same chirality, corresponding to $(N, 0)$ supersymmetry. The $R$-symmetry group is then $\operatorname{Sp}(N)$ and the corresponding superspace is $\mathbb{R}^{6 \mid 8 N}$. We define superinversion as a map to the associated superspace of opposite chirality. General formulae for two-point and three-point correlation functions of quasi-primary superfields are exhibited. The superconformal group in six-dimensions is reduced to a corresponding extended superconformal group in four-dimensions. Superconformally covariant differential operators are also discussed.
\end{abstract}

\footnotetext{
*E-mail address: J.H.Park@damtp.cam.ac.uk
} 


\section{Introduction}

Theories with extended supersymmetry in four-dimensions may have a close relation to simpler theories in higher dimensions, as dimensional reduction of the latter often leads to the former. In particular, reductions of the six and ten dimensional $N=1$ supersymmetric theories yield $N=2$ and $N=4$ supersymmetric theories in four-dimensions. Six-dimensional supersymmetric theories have been studied some time ago by various authors, e.g. [1 [5]. More recently there is evidence from $\mathrm{M}$ /string theory for the presence of renormalization group fixed points in six-dimensions and hence the existence of non-trivial six-dimensional theories with superconformal invariance; see [6,7] and references given there. It remains an open problem to construct explicitly six-dimensional superconformal theories in a superspace formalism, although some actions have been proposed in terms of component fields

- scalar, spinor and two-form tensor fields 8, 9] such that the three-form formed by its exterior derivative is self-dual. Note that in six-dimensions the self-duality condition is a real constraint with Minkowskian signature and theories with a two-form field may be conformal, since, in general, under conformal transformations the exterior derivative of $p$-form transforms homogeneously only in $d=2(p+1)$ dimensions.

It is well known that conformal transformations may be defined for spacetimes of any dimension. However, not all spacetime dimensions allow the corresponding supersymmetry algebra to be extended to a superconformal algebra. All possible superconformal algebras were analyzed by Nahm [10] based on the classification of real simple Lie superalgebras [11]. According to [10] the standard supersymmetry algebra admits an extension to a superconformal algebra only if $d \leq 6$. In particular, the highest dimension admitting superconformal algebra is six and in $d=4,6$ dimensions, which are of our interest in this paper, the bosonic part of the superconformal algebra is

$$
\begin{array}{ll}
d=4 ; & \mathrm{o}(4,2) \oplus \mathrm{u}(N), N \neq 4 \quad \text { or } \mathrm{o}(4,2) \oplus \mathrm{su}(4) \\
d=6 ; & \mathrm{o}(6,2) \oplus \mathrm{sp}(N)
\end{array}
$$

On six-dimensional Minkowskian spacetime it is possible to define Weyl spinors of opposite chiralities and so the six-dimensional supersymmetry may be denoted by two numbers, $(N, \tilde{N})$, where $N$ and $\tilde{N}$ are the numbers of chiral and anti-chiral supercharges. The $R$ symmetry group is then $\operatorname{Sp}(N) \times \operatorname{Sp}(\tilde{N})$. The analysis of Nahm shows that to admit a superconformal algebra either $N$ or $\tilde{N}$ should be zero. Although both $(1,1)$ and $(2,0)$ supersymmetry give rise $N=4$ four-dimensional supersymmetry after dimensional reduction, only $(2,0)$ supersymmetry theories can be superconformal [12].

The above analysis is essentially based on the classification of real simple Lie superalgebras and identification of the bosonic part with the usual spacetime conformal symmetry. This 
approach does not rely on any definition of superconformal transformation in superspace. In fact, by analyzing possible forms of the commutators between conformal symmetry and supersymmetry and ensuring compatibility with the Jacobi identity, the six-dimensional superconformal algebra may be constructed [9].

In this paper, we analyze six-dimensional superconformal symmetry directly in terms of coordinate transformations on superspace. The superconformal group is independently derived and we also obtain concise formulae for correlation functions of superfields which transform simply under superconformal transformations. This approach has previously been applied in four-dimensions; for the analysis of superconformally invariant correlation functions in four-dimensions see our earlier work [13] which follows analogous discussions in the non supersymmetric case [14, 15]. General discussions of superconformal invariance in four spacetime dimensions are contained in [16, 17] which have further references.

The organization of this paper is as follows. In section 2 , we review six-dimensional $(N, \tilde{N})$ supersymmetry. For six-dimensional Weyl spinors a pseudo-Majorana notation [18] is introduced, which simplifies the formalism significantly. In section 3, we first define the six-dimensional superconformal group in terms of superspace coordinate transformations as a generalization of the definition of ordinary conformal transformations. We then derive a sufficient and necessary condition for a supercoordinate transformation to be superconformal. The general solutions are identified in terms of supertranslations, dilations, Lorentz transformations, $R$-symmetry transformations and special superconformal transformations for $(N, 0)$ supersymmetry. On the other hand, if $N, \tilde{N}>0$, we show that although scale transformations may be introduced, there exist no special superconformal transformations as expected from Nahm's result. We also define superinversion as a map from $(N, 0)$ superspace to $(0, N)$ superspace. The $(N, 0)$ superconformal group is identified with a supermatrix group, $\operatorname{OSp}(2,6 \mid N)$ having dimensions $(28+N(2 N+1) \mid 16 N)$. In section 4, we obtain an explicit formula for the finite superconformal transformations of the supercoordinates. We also discuss about functions depending on two or three points in superspace which transform covariantly under superconformal transformations. These functions are crucial to obtain two and three points correlation functions later. In section 5 , the $(N, 0)$ superconformal group in six-dimensions is reduced to a corresponding superconformal group in four-dimensions. In particular, the $R$-symmetry group is reduced from $\operatorname{Sp}(N)$ to $\mathrm{U}(N)$ if $N \neq 4$ and $\mathrm{SU}(4)$ if $N=4$, as in eq.(1.1). In section 6, we discuss the conditions of superconformal invariance for correlation functions for quasi-primary superfields. General forms of two-point and three-point functions are exhibited and explicit formulae for twopoint functions of superfields in simple cases are given as well. In an appendix, some useful equations and $(N, 0)$ superconformal algebra are given. Superconformally covariant differential operators are also discussed by considering the conditions for superfields formed by the action of spinor derivatives on quasi-primary superfields to remain quasi-primary. 


\section{Supersymmetry in Six-dimensions}

With the six-dimensional Minkowskian metric $\eta^{A B}=\operatorname{diag}(+1,-1,-1 \cdots-1)$, the $8 \times 8$ gamma matrices, $\Gamma^{A}, A=0,1, \cdots 5$, satisfy the Clifford algebra

$$
\Gamma^{A} \Gamma^{B}+\Gamma^{B} \Gamma^{A}=2 \eta^{A B}
$$

The gamma matrices for even dimensions can be chosen in general to have the form

$$
\Gamma^{A}=\left(\begin{array}{cc}
0 & \gamma^{A} \\
\tilde{\gamma}^{A} & 0
\end{array}\right)
$$

where the generalization of $\gamma_{5}$ is diagonal

$$
\Gamma^{7}=\Gamma^{0} \Gamma^{1} \Gamma^{2} \Gamma^{3} \Gamma^{4} \Gamma^{5}=\left(\begin{array}{cc}
1 & 0 \\
0 & -1
\end{array}\right)
$$

We also assume the hermiticity condition

$$
\Gamma^{0} \Gamma^{A \dagger} \Gamma^{0}=\Gamma^{A}
$$

The $4 \times 4$ matrices, $\gamma^{A}, \tilde{\gamma}^{A}$ satisfy from eq.(2.1)

$$
\gamma^{A} \tilde{\gamma}^{B}+\gamma^{B} \tilde{\gamma}^{A}=2 \eta^{A B}
$$

and

$$
\operatorname{tr}\left(\gamma^{A} \tilde{\gamma}^{B}\right)=4 \eta^{A B}
$$

From eq.(2.4) we have

$$
\gamma^{0} \gamma^{A \dagger} \gamma^{0}=\gamma^{A} \quad \gamma^{A \dagger}=\tilde{\gamma}_{A}
$$

In six-dimensions, $\gamma^{A}, \tilde{\gamma}^{A}$ may also be taken to be anti-symmetric

$$
\left(\gamma^{A}\right)_{\alpha \beta}=-\left(\gamma^{A}\right)_{\beta \alpha} \quad\left(\tilde{\gamma}^{A}\right)^{\alpha \beta}=-\left(\tilde{\gamma}^{A}\right)^{\beta \alpha}
$$

$\left\{\gamma^{A}\right\}$ and $\left\{\tilde{\gamma}^{A}\right\}$ are separately bases of $4 \times 4$ anti-symmetric matrices so that

$$
\left(\gamma^{A}\right)_{\alpha \beta}\left(\tilde{\gamma}_{A}\right)^{\gamma \delta}=2\left(\delta_{\alpha}^{\delta} \delta_{\beta}^{\gamma}-\delta_{\alpha}^{\gamma} \delta_{\beta}^{\delta}\right)
$$

with the coefficient determined by eq.(2.6).

$\left\{\gamma^{[A} \tilde{\gamma}^{B]}, 1\right\}$ also forms a basis of general $4 \times 4$ matrices with the completeness relation

$$
-\frac{1}{8}\left(\gamma^{[A} \tilde{\gamma}^{B]}\right)_{\alpha}^{\beta}\left(\gamma_{[A} \tilde{\gamma}_{B]}\right)_{\gamma}^{\delta}+\frac{1}{4} \delta_{\alpha}^{\beta} \delta_{\gamma}{ }^{\delta}=\delta_{\alpha} \delta_{\gamma}{ }^{\beta}
$$


With this choice of gamma matrices we get巴

$$
\begin{aligned}
& \left(\gamma^{A}\right)_{\alpha \beta}=\frac{1}{2} \epsilon_{\alpha \beta \gamma \delta}\left(\tilde{\gamma}^{A}\right)^{\gamma \delta} \\
& \left(\tilde{\gamma}^{A}\right)^{\alpha \beta}=\frac{1}{2} \epsilon^{\alpha \beta \gamma \delta}\left(\gamma^{A}\right)_{\gamma \delta}
\end{aligned}
$$

The supersymmetry algebra has the standard form

$$
\left\{\mathcal{Q}^{a}, \overline{\mathcal{Q}}_{b}\right\}=2 \delta^{a}{ }_{b} \Gamma^{A} P_{A}
$$

The eight-component spinorial supercharge, $\mathcal{Q}^{a}$ and Grassman variable, $\vartheta^{a}(a=1,2, \cdots N)$ can be written in the basis given by eqs. 22.2, 2.3.

$$
\begin{aligned}
\mathcal{Q}^{a}=\left(\begin{array}{c}
\mathcal{Q}_{+}^{a} \\
\mathcal{Q}_{-}^{a}
\end{array}\right) & \overline{\mathcal{Q}}_{a}=\mathcal{Q}^{a \dagger} \Gamma^{0}=\left(\overline{\mathcal{Q}}_{a}^{-}, \overline{\mathcal{Q}}_{a}^{+}\right) \\
\vartheta^{a}=\left(\begin{array}{c}
\vartheta_{+}^{a} \\
\vartheta_{-}^{a}
\end{array}\right) & \bar{\vartheta}_{a}=\vartheta^{a \dagger} \Gamma^{0}=\left(\bar{\vartheta}_{a}^{-}, \bar{\vartheta}_{a}^{+}\right)
\end{aligned}
$$

where the superscripts/subscripts \pm denote Weyl spinors.

With the decomposition (2.13) and (2.14) we define for $1 \leq i \leq 2 N$

$$
\begin{array}{rlrl}
Q^{i} & =\left(\begin{array}{c}
\mathcal{Q}_{+}^{a} \\
\left(\overline{\mathcal{Q}}_{a}^{+}\right)^{t}
\end{array}\right) & \tilde{Q}^{i}=\left(\begin{array}{c}
\mathcal{Q}_{-}^{a} \\
\left(\overline{\mathcal{Q}}_{a}^{-}\right)^{t}
\end{array}\right) \\
\theta^{i}=\left(\begin{array}{c}
\vartheta_{-}^{a} \\
\left(\bar{\vartheta}_{a}^{-}\right)^{t}
\end{array}\right) & \tilde{\theta}^{i}=\left(\begin{array}{c}
\vartheta_{+}^{a} \\
\left(\bar{\vartheta}_{a}^{+}\right)^{t}
\end{array}\right)
\end{array}
$$

With these definitions the supersymmetry algebra (2.12) is equivalent in six-dimensions to

$$
\begin{aligned}
& \left\{Q^{i},\left(Q^{j}\right)^{t}\right\}=2 \mathcal{E}^{i j} \gamma^{A} P_{A} \\
& \left\{\tilde{Q}^{i},\left(\tilde{Q}^{j}\right)^{t}\right\}=2 \mathcal{E}^{i j} \tilde{\gamma}^{A} P_{A}
\end{aligned}
$$

where $\mathcal{E}^{i j}$ is

$$
\mathcal{E}^{i j}=\left(\begin{array}{cc}
0 & \delta^{a}{ }_{b} \\
-\delta_{a}{ }^{b} & 0
\end{array}\right)
$$

and the inverse, $\overline{\mathcal{E}}_{i j}$, is defined by $\mathcal{E}^{i j} \overline{\mathcal{E}}_{j k}=\delta^{i}{ }_{k}$

$$
\overline{\mathcal{E}}_{j k}=\left(\begin{array}{cc}
0 & -\delta_{b}^{c} \\
\delta_{c}^{b} & 0
\end{array}\right)
$$

\footnotetext{
${ }^{1}$ We put $\epsilon_{1234}=\epsilon^{1234}=1$. See Appendix A for further discussion.
} 
$Q^{i}, \theta^{i}, \tilde{Q}^{i}, \tilde{\theta}^{i}$ satisfy the pseudo-Majorana conditions

$$
\begin{aligned}
\bar{Q}_{i}=Q^{i \dagger} \gamma^{0}=\left(Q^{j}\right)^{t} \overline{\mathcal{E}}_{j i} & \overline{\tilde{Q}}_{i}=\tilde{Q}^{i \dagger} \tilde{\gamma}^{0}=\left(\tilde{Q}^{j}\right)^{t} \overline{\mathcal{E}}_{j i} \\
\bar{\theta}_{i}=\theta^{i \dagger} \tilde{\gamma}^{0}=\left(\theta^{j}\right)^{t} \overline{\mathcal{E}}_{j i} & \overline{\tilde{\theta}}_{i}=\tilde{\theta}^{i \dagger} \gamma^{0}=\left(\tilde{\theta}^{j}\right)^{t} \overline{\mathcal{E}}_{j i}
\end{aligned}
$$

which give

$$
\begin{gathered}
\bar{\vartheta}_{a} \mathcal{Q}^{a}+\overline{\mathcal{Q}}_{a} \vartheta^{a}=\bar{Q}_{i} \theta^{i}+\overline{\tilde{Q}}_{i} \tilde{\theta}^{i} \\
\bar{Q}_{i} \theta^{i}=\bar{\theta}_{i} Q^{i} \quad \overline{\tilde{Q}}_{i} \tilde{\theta}^{i}=\overline{\tilde{\theta}}_{i} \tilde{Q}^{i}
\end{gathered}
$$

The six-dimensional supersymmetry algebra obtained here can be generalized by replacing eq.(2.17b) by

$$
\left\{\tilde{Q}^{\tilde{\imath}},\left(\tilde{Q}^{\tilde{\jmath}}\right)^{t}\right\}=2 \tilde{\mathcal{E}}^{\tilde{\imath} \tilde{\jmath}} \tilde{\gamma}^{A} P_{A} \quad \tilde{\imath}, \tilde{\jmath}=1,2, \cdots, 2 \tilde{N}
$$

with $\tilde{\mathcal{E}}$, the appropriate anti-symmetric matrix, so that with eq.(2.17a) they form the sixdimensional $(N, \tilde{N})$ supersymmetry algebra. The above discussion therefore demonstrates that the supersymmetry algebra (2.12) corresponds to $(N, N)$ supersymmetry.

The exterior derivative, $\mathrm{d}$, on superspace with coordinates $z^{\mathcal{A}}=\left(x^{A}, \theta^{i \alpha}, \tilde{\theta}_{\alpha}^{\tilde{\imath}}\right)$, is defined as

$$
\mathrm{d} \equiv \mathrm{d} z^{\mathcal{A}} \frac{\partial}{\partial z^{\mathcal{A}}}=e^{\mathcal{A}} D_{\mathcal{A}}=e^{A} \partial_{A}+\mathrm{d} \theta^{i \alpha} D_{i \alpha}+\mathrm{d} \tilde{\theta}_{\alpha}^{\tilde{\imath}} \tilde{D}_{\tilde{\imath}}^{\alpha}
$$

where $e^{\mathcal{A}}=\left(e^{A}, \mathrm{~d} \theta^{i \alpha}, \mathrm{d} \tilde{\theta}_{\alpha}^{\tilde{\imath}}\right)$ are supertranslation invariant one forms

$$
e^{A}(z)=\mathrm{d} x^{A}-i \bar{\theta}_{i} \gamma^{A} \mathrm{~d} \theta^{i}-i \overline{\tilde{\theta}}_{\tilde{\imath}} \tilde{\gamma}^{A} \mathrm{~d} \tilde{\theta}^{\tilde{\imath}}
$$

and $D_{\mathcal{A}}=\left(\partial_{A}, D_{i \alpha}, \tilde{D}_{\tilde{\imath}}^{\alpha}\right)$ are covariant derivatives

$$
\partial_{A}=\frac{\partial}{\partial x^{A}} \quad D_{i \alpha}=\frac{\partial}{\partial \theta^{i \alpha}}-i\left(\bar{\theta}_{i} \gamma^{A}\right)_{\alpha} \frac{\partial}{\partial x^{A}} \quad \tilde{D}_{\tilde{\imath}}^{\alpha}=\frac{\partial}{\partial \tilde{\theta}_{\alpha}^{\tilde{\imath}}}-i\left(\overline{\tilde{\theta}}_{\tilde{\imath}} \tilde{\gamma}^{A}\right)^{\alpha} \frac{\partial}{\partial x^{A}}
$$

satisfying the anti-commutator relations

$$
\left\{D_{i \alpha}, D_{j \beta}\right\}=-2 i \overline{\mathcal{E}}_{i j} \gamma_{\alpha \beta}^{A} \partial_{A} \quad\left\{\tilde{D}_{\tilde{\imath}}^{\alpha}, \tilde{D}_{\tilde{\jmath}}^{\beta}\right\}=-2 i \overline{\tilde{\mathcal{E}}}_{\tilde{\imath} \tilde{\jmath}} \tilde{\gamma}^{A \alpha \beta} \partial_{A}
$$

Under an arbitrary superspace coordinate transformation, $z \rightarrow z^{\prime}, e^{\mathcal{A}}$ and $D_{\mathcal{A}}$ transform as

$$
e^{\mathcal{A}}\left(z^{\prime}\right)=e^{\mathcal{B}}(z) \mathcal{R}_{\mathcal{B}}{ }^{\mathcal{A}}(z) \quad D_{\mathcal{A}}^{\prime}=\mathcal{R}^{-1}{ }_{\mathcal{A}}^{\mathcal{B}}(z) D_{\mathcal{B}}
$$

so that the exterior derivative is left invariant

$$
e^{\mathcal{A}}(z) D_{\mathcal{A}}=e^{\mathcal{A}}\left(z^{\prime}\right) D_{\mathcal{A}}^{\prime}
$$




\section{Superconformal Symmetry in Six-dimensions}

The superconformal group is defined here as a group of superspace coordinate transformations, $z \stackrel{g}{\longrightarrow} z^{\prime}$ that preserve the infinitesimal supersymmetric interval length, $e^{2}=\eta_{A B} e^{A} e^{B}$ upto a local scale factor, so that

$$
e^{2}(z) \rightarrow e^{2}\left(z^{\prime}\right)=\Omega^{2}(z ; g) e^{2}(z)
$$

where $\Omega(z ; g)$ is a local scale factor.

This requires

$$
e^{A}\left(z^{\prime}\right)=e^{B}(z) R_{B}^{A}(z ; g)
$$

and

$$
R_{A}^{C}(z ; g) R_{B}^{D}(z ; g) \eta_{C D}=\Omega^{2}(z ; g) \eta_{A B}
$$

giving

$$
\operatorname{det} R(z ; g)=\Omega^{6}(z ; g)
$$

To ensure eq.(3.2), $\mathcal{R}_{\mathcal{A}}{ }^{\mathcal{B}}(z ; g)$ in eq.(2.29) must be of the form

$$
\mathcal{R}_{\mathcal{A}}^{\mathcal{B}}(z ; g)=\left(\begin{array}{ccc}
R_{A}^{B}(z ; g) & \partial_{A} \theta^{\prime j \beta} & \partial_{A} \tilde{\theta}_{\beta}^{\prime \tilde{j}} \\
0 & D_{i \alpha} \theta^{\prime j \beta} & D_{i \alpha} \tilde{\theta}_{\beta}^{\prime \prime} \\
0 & \tilde{D}_{\tilde{\imath}}^{\alpha} \theta^{\prime j \beta} & \tilde{D}_{\tilde{\imath}}^{\alpha} \tilde{\theta}_{\beta}^{\prime j}
\end{array}\right)
$$

To achieve the form (3.5) we must impose

$$
\begin{aligned}
& D_{i \alpha} x^{\prime A}+i \bar{\theta}_{j}^{\prime} \gamma^{A} D_{i \alpha} \theta^{\prime j}+i \overline{\tilde{\theta}}_{\tilde{\jmath}}^{\prime} \tilde{\gamma}^{A} D_{i \alpha} \tilde{\theta}^{\prime \tilde{\jmath}}=0 \\
& \tilde{D}_{\tilde{\imath}}^{\alpha} x^{\prime A}+i \bar{\theta}_{j}^{\prime} \gamma^{A} \tilde{D}_{\tilde{i}}^{\alpha} \theta^{\prime j}+i \overline{\tilde{\theta}}_{\tilde{j}}^{\prime} \tilde{\gamma}^{A} \tilde{D}_{\tilde{\imath}}^{\alpha} \tilde{\theta}^{\prime \tilde{\jmath}}=0
\end{aligned}
$$

while $R_{A}{ }^{B}$ is given by

$$
R_{A}^{B}(z ; g)=\frac{\partial x^{\prime B}}{\partial x^{A}}-i \bar{\theta}_{i}^{\prime} \gamma^{B} \frac{\partial \theta^{\prime i}}{\partial x^{A}}-i \overline{\tilde{\theta}}_{\tilde{\imath}}^{\prime} \tilde{\gamma}^{B} \frac{\partial \tilde{\theta}^{\prime \tilde{\imath}}}{\partial x^{A}}
$$

and $R(z ; g)$ must also satisfy eq.(3.3). $R(z ; g)$ forms a representation of the superconformal group, since under the successive superconformal transformations, $z \stackrel{g}{\longrightarrow} z^{\prime} \stackrel{g^{\prime}}{\longrightarrow} z^{\prime \prime}$ giving $z \stackrel{g^{\prime \prime}}{\longrightarrow} z^{\prime \prime}$, we have

$$
R(z ; g) R\left(z^{\prime} ; g^{\prime}\right)=R\left(z ; g^{\prime \prime}\right)
$$


Infinitesimally $z^{\prime} \simeq z+\delta z$, eq.(3.6a) gives, if $N>0$,

$$
D_{i \alpha} h^{A}=-2 i\left(\bar{\lambda}_{i} \gamma^{A}\right)_{\alpha}
$$

and for $\tilde{N}>0$

$$
\tilde{D}_{\tilde{\imath}}^{\alpha} h^{A}=-2 i\left(\overline{\tilde{\lambda}}_{\tilde{\imath}} \tilde{\gamma}^{A}\right)^{\alpha}
$$

where we define

$$
\begin{gathered}
\lambda^{i}=\delta \theta^{i} \quad \tilde{\lambda}^{\tilde{\imath}}=\delta \tilde{\theta}^{\tilde{\imath}} \\
h^{A}=\delta x^{A}-i \bar{\theta}_{i} \gamma^{A} \delta \theta^{i}-i \overline{\tilde{\theta}}_{\tilde{\imath}} \tilde{\gamma}^{A} \delta \tilde{\theta}^{\tilde{\imath}}
\end{gathered}
$$

Infinitesimally $R_{A}^{B}$ from eq.(3.7) is of the form

$$
R_{A}^{B} \simeq \delta_{A}^{B}+\partial_{A} h^{B}
$$

so that the condition (3.3) reduces to the ordinary conformal Killing equation

$$
\partial_{A} h_{B}+\partial_{B} h_{A} \propto \eta_{A B}
$$

This result follows from eq.(3.9), since using the anti-commutator relation for $D_{i \alpha}(2.28)$ we get from eq.(3.9)

$$
\overline{\mathcal{E}}_{i j} \partial_{B} h^{A}=\frac{1}{4}\left(D_{i \alpha}\left(\bar{\lambda}_{j} \gamma^{A} \tilde{\gamma}_{B}\right)^{\alpha}-D_{j \alpha}\left(\bar{\lambda}_{i} \gamma^{A} \tilde{\gamma}_{B}\right)^{\alpha}\right)
$$

and hence

$$
\overline{\mathcal{E}}_{i j}\left(\partial_{A} h_{B}+\partial_{B} h_{A}\right)=\frac{1}{2}\left(D_{i \alpha} \bar{\lambda}_{j}^{\alpha}-D_{j \alpha} \bar{\lambda}_{i}^{\alpha}\right) \eta_{A B}
$$

which implies

$$
\begin{aligned}
& \partial_{A} h_{B}+\partial_{B} h_{A}=2 \rho \eta_{A B} \\
& D_{i \alpha} \bar{\lambda}_{j}^{\alpha}-D_{j \alpha} \bar{\lambda}_{i}^{\alpha}=4 \rho \overline{\mathcal{E}}_{i j}
\end{aligned}
$$

A similar equation may also be obtained from eq.(3.10).

Hence eqs. (3.9, 3.10) are sufficient and necessary conditions for $(N, \tilde{N})$ superconformal symmetry, although if $\tilde{N}=0$ eq.(3.9) alone is sufficient. 2

\footnotetext{
${ }^{2}$ While this work was being completed, similar equations to (3.9, 3.14 were discussed by Grojean and Mourad [19].
} 
In six-dimensions there is a unique correspondence between a general six vector, $v^{A}$, and an anti-symmetric matrix, $\mathrm{v}_{\alpha \beta}$ or $\tilde{\mathrm{v}}^{\alpha \beta}$ through

$$
\begin{array}{rlrl}
\mathrm{v}_{\alpha \beta} & =v^{A} \gamma_{A \alpha \beta} & v^{A}=\frac{1}{4} \operatorname{tr}\left(\tilde{\gamma}^{A} \mathrm{v}\right) \\
\tilde{\mathrm{v}}^{\alpha \beta}=v^{A} \tilde{\gamma}_{A}^{\alpha \beta} & v^{A}=\frac{1}{4} \operatorname{tr}\left(\gamma^{A} \tilde{\mathrm{v}}\right)
\end{array}
$$

where

$$
\mathrm{v}_{\alpha \beta}=\frac{1}{2} \epsilon_{\alpha \beta \gamma \delta} \tilde{\mathrm{v}}^{\gamma \delta} \quad \tilde{\mathrm{v}}^{\alpha \beta}=\frac{1}{2} \epsilon^{\alpha \beta \gamma \delta} \mathrm{v}_{\gamma \delta}
$$

With this notation and using eq.(2.9), eq.(3.9) is equivalent to

$$
D_{i \alpha} \tilde{\mathrm{h}}^{\beta \gamma}=\frac{1}{3}\left(\delta_{\alpha}^{\beta} D_{i \delta} \tilde{\mathrm{h}}^{\delta \gamma}-\delta_{\alpha}^{\gamma} D_{i \delta} \tilde{\mathrm{h}}^{\delta \beta}\right)
$$

and $\bar{\lambda}_{i}^{\alpha}$ is given by

$$
\bar{\lambda}_{i}^{\alpha}=i \frac{1}{12} D_{i \beta} \tilde{\mathrm{h}}^{\beta \alpha}
$$

Eq.(3.20) may therefore be regarded as the fundamental $(N, 0)$ superconformal Killing equation.

To solve these equations we first write a general solution of eq.(3.14) as

$$
h^{A}(z)=a^{A}(\theta)+\lambda(\theta) x^{A}+w_{B}^{A}(\theta) x^{B}+2 x \cdot b(\theta) x^{A}-x^{2} b^{A}(\theta)
$$

where $w_{A B}(\theta)+w_{B A}(\theta)=0$. It is convenient to introduce the variables

$$
\tilde{\mathrm{x}}_{ \pm}=\tilde{\mathrm{x}} \pm 2 i \theta^{i} \bar{\theta}_{i}
$$

where

$$
\tilde{\mathrm{x}}_{ \pm}^{t}=-\tilde{\mathrm{x}}_{\mp} \quad \tilde{\mathrm{x}}_{ \pm}=\tilde{\gamma}^{0} \tilde{\mathrm{x}}_{\mp}^{\dagger} \tilde{\gamma}^{0}
$$

Then eq.(3.22) can be written in terms of $\tilde{\mathrm{h}}=h^{A} \tilde{\gamma}_{A}$ as

$$
\tilde{\mathrm{h}}(z)=\tilde{\mathrm{x}}_{-} \mathrm{b}(\theta) \tilde{\mathrm{x}}_{+}+W(\theta) \tilde{\mathrm{x}}_{+}+\tilde{\mathrm{x}}_{-} W(\theta)^{t}+\tilde{\mathrm{A}}(\theta)
$$

where

$$
\begin{gathered}
W(\theta)=\frac{1}{4} w_{A B}(\theta) \tilde{\gamma}^{A} \gamma^{B}+\frac{1}{2} \lambda(\theta)+2 i \theta^{i} \bar{\theta}_{i} \mathrm{~b}(\theta) \\
\tilde{\mathrm{A}}(\theta)=\tilde{\mathrm{a}}(\theta)+i \frac{1}{2} w_{A B}(\theta)\left(\theta^{i} \bar{\theta}_{i} \gamma^{B} \tilde{\gamma}^{A}-\tilde{\gamma}^{A} \gamma^{B} \theta^{i} \bar{\theta}_{i}\right)+4\left(\bar{\theta}_{i} \mathrm{~b}(\theta) \theta^{j}\right) \theta^{i} \bar{\theta}_{j}=-(\tilde{\mathrm{A}}(\theta))^{t}
\end{gathered}
$$

Essentially we may regard $W(\theta)$ as an arbitrary $4 \times 4$ matrix and $\tilde{\mathrm{A}}(\theta)$ as an arbitrary $4 \times 4$ anti-symmetric matrix.

The variables, $\tilde{\mathrm{x}}_{ \pm}$defined in eq.(3.23), satisfy

$$
D_{i \alpha} \tilde{\mathrm{x}}_{+}^{\beta \gamma}=4 i \delta_{\alpha}^{\gamma} \bar{\theta}_{i}^{\beta} \quad D_{i \alpha} \tilde{\mathrm{x}}_{-}^{\beta \gamma}=-4 i \delta_{\alpha}{ }^{\beta} \bar{\theta}_{i}^{\gamma}
$$


which ensures that substituting eq. (3.25) into eq. (3.20) leads independent equations for $\mathrm{b}(\theta), W(\theta), \tilde{\mathrm{A}}(\theta)$. Here, we outline the method of solution.]

Considering the $x^{2}$-terms one can show

$$
D_{i \alpha} \mathrm{b}(\theta)=0
$$

and so $b^{A}(\theta)$ is independent of $\theta$ and hence eq.(3.28) shows that $\tilde{\mathrm{x}}_{-} \mathrm{b} \tilde{\mathrm{x}}_{+}$is a solution of eq. (3.20). Now focusing on the linear terms in $x$, one can show

$$
\begin{gathered}
D_{i \alpha} W_{\gamma}^{\beta}(\theta)=\delta_{\alpha}^{\beta} D_{i \gamma} W_{\delta}^{\delta}(\theta) \\
D_{i \alpha} D_{j \beta} W_{\delta}^{\gamma}(\theta)=0
\end{gathered}
$$

These two relations determine $W(\theta)$

$$
W(\theta)=-4 \theta^{i} \bar{\rho}_{i}+\omega+\frac{1}{2} \lambda \quad W(\theta)^{t}=-4 \rho^{i} \bar{\theta}_{i}-\tilde{\omega}+\frac{1}{2} \lambda
$$

where for $\omega_{A B}=-\omega_{B A}$

$$
\omega=\frac{1}{4} \omega_{A B} \tilde{\gamma}^{A} \gamma^{B} \quad \tilde{\omega}=\frac{1}{4} \omega_{A B} \gamma^{A} \tilde{\gamma}^{B}
$$

and $\rho_{\alpha}^{i}, \bar{\rho}_{i \alpha}$ satisfy

$$
\bar{\rho}_{i}=\left(\rho^{j}\right)^{t} \overline{\mathcal{E}}_{j i}
$$

$W(\theta) \tilde{\mathbf{x}}_{+}+\tilde{\mathrm{x}}_{-} W(\theta)^{t}$ is a solution of eq.(3.20) as well. Therefore the remaining terms are

$$
D_{i \alpha} \tilde{\mathrm{A}}^{\beta \gamma}(\theta)=\frac{1}{3}\left(\delta_{\alpha}^{\beta} D_{i \delta} \tilde{\mathrm{A}}^{\delta \gamma}(\theta)-\delta_{\alpha}^{\gamma} D_{i \delta} \tilde{\mathrm{A}}^{\delta \beta}(\theta)\right)
$$

from which one can derive

$$
\begin{gathered}
D_{i \alpha} D_{j \beta} \tilde{\mathrm{A}}^{\gamma \delta}(\theta)=\frac{1}{12}\left(\delta_{\alpha}^{\gamma} \delta_{\beta}{ }^{\delta}-\delta_{\alpha}{ }^{\delta} \delta_{\beta}{ }^{\gamma}\right) D_{i \epsilon} D_{j \eta} \tilde{\mathrm{A}}^{\epsilon \eta}(\theta) \\
D_{i \alpha} D_{j \beta} D_{k \gamma} \tilde{\mathrm{A}}(\theta)=0
\end{gathered}
$$

and so $\tilde{\mathrm{A}}(\theta)$ is at most quadratic in $\theta$. By virtue of these two equations, it is straightforward to get the following form of $\tilde{\mathrm{A}}(\theta)$

$$
\tilde{\mathrm{A}}(\theta)=-4 i \theta^{i} T_{i}{ }^{j} \bar{\theta}_{j}+4 i\left(\varepsilon^{i} \bar{\theta}_{i}-\theta^{i} \bar{\varepsilon}_{i}\right)+\tilde{\mathrm{a}}
$$

where $T_{i}^{j}, \varepsilon^{i \alpha}, \bar{\varepsilon}_{i}^{\alpha}$ satisfy

$$
\begin{gathered}
T^{t} \mathcal{E}+\mathcal{E} T=0 \\
\bar{\varepsilon}_{i}=\left(\varepsilon^{j}\right)^{t} \overline{\mathcal{E}}_{j i}
\end{gathered}
$$

\footnotetext{
${ }^{3}$ The details of deriving eqs. 3.29, 3.30, 3.31, 3.36, 3.37 can be found in Appendix B.
} 
Each term appearing in eq.(3.38) is an independent solution of eq.(3.20).

All together, we now have the following general solution of eq.(3.20)

$$
\tilde{\mathrm{h}}=\tilde{\mathrm{x}}_{-} \mathrm{b} \tilde{\mathrm{x}}_{+}+\left(\omega+\frac{1}{2} \lambda-4 \theta^{i} \bar{\rho}_{i}\right) \tilde{\mathrm{x}}_{+}-\tilde{\mathrm{x}}_{-}\left(\tilde{\omega}-\frac{1}{2} \lambda+4 \rho^{i} \bar{\theta}_{i}\right)-4 i \theta^{i} T_{i}^{j} \bar{\theta}_{j}+4 i\left(\varepsilon^{i} \bar{\theta}_{i}-\theta^{i} \bar{\varepsilon}_{i}\right)+\tilde{\mathrm{a}}
$$

which induces

$$
\lambda^{i}(z)=\varepsilon^{i}+\frac{1}{2} \lambda \theta^{i}+\omega \theta^{i}-\theta^{j} T_{j}^{i}+\tilde{\mathrm{x}}_{-} \mathrm{b} \theta^{i}+i \tilde{\mathrm{x}}_{-} \rho^{i}-4\left(\bar{\rho}_{j} \theta^{i}\right) \theta^{j}
$$

We may also rewrite the result $(3.40)$ as

$$
\begin{aligned}
& \delta \tilde{\mathrm{x}}_{+}=\tilde{\mathrm{x}}_{+} \mathrm{b} \tilde{\mathrm{x}}_{+}-4 \tilde{\mathrm{x}}_{+} \rho^{i} \bar{\theta}_{i}+\lambda \tilde{\mathrm{x}}_{+}+\omega \tilde{\mathrm{x}}_{+}-\tilde{\mathrm{x}}_{+} \tilde{\omega}+4 i \varepsilon^{i} \bar{\theta}_{i}+\tilde{\mathrm{a}} \\
& \delta \tilde{\mathrm{x}}_{-}=\tilde{\mathrm{x}}_{-} \mathrm{b} \tilde{\mathrm{x}}_{-}-4 \theta^{i} \bar{\rho}_{i} \tilde{\mathrm{x}}_{-}+\lambda \tilde{\mathrm{x}}_{-}+\omega \tilde{\mathrm{x}}_{-}-\tilde{\mathrm{x}}_{-} \tilde{\omega}-4 i \theta^{i} \bar{\varepsilon}_{i}+\tilde{\mathrm{a}}
\end{aligned}
$$

Furthermore, imposing the reality condition for $x^{A}$ and $\bar{\theta}_{i}=\theta^{i \dagger} \tilde{\gamma}^{0}$ gives the following extra conditions

$$
\begin{gathered}
a^{A *}=a^{A} \quad b^{A *}=b^{A} \quad \omega_{A B}{ }^{*}=\omega_{A B} \quad \lambda^{*}=\lambda \quad T^{\dagger}=-T \\
\bar{\rho}_{i}=\rho^{i \dagger} \gamma^{0} \quad \bar{\varepsilon}_{i}=\varepsilon^{i \dagger} \tilde{\gamma}^{0}
\end{gathered}
$$

The solutions we obtained here read the generators of $(N, 0)$ superconformal transformations.

\section{$3.1(N, 0)$ Superconformal Transformations}

In summary the generators of $(N, 0)$ superconformal transformations in six-dimensions acting on the six-dimensional chiral superspace, $\mathbb{R}_{+}^{6 \mid 8 N}$, with coordinates, $z^{\mathcal{A}}=\left(x^{A}, \theta^{i}\right)$ can be reduced to

1. Supertranslations

$$
\delta x^{A}=a^{A}-i \bar{\theta}_{i} \gamma^{A} \varepsilon^{i} \quad \delta \theta^{i}=\varepsilon^{i}
$$

2. Dilations

$$
\delta x^{A}=\lambda x^{A} \quad \delta \theta^{i}=\frac{1}{2} \lambda \theta^{i}
$$

3. Lorentz transformations, with $\omega$ defined in eq.(3.33),

$$
\delta x^{A}=\omega_{B}^{A} x^{B} \quad \delta \theta^{i}=\omega \theta^{i}
$$


4. $R$-symmetry transformations belonging to $\operatorname{Sp}(N)$, of dimension $N(2 N+1)$,

$$
\delta x^{A}=0 \quad \delta \theta^{i}=-\theta^{j} T_{j}^{i}
$$

where the $2 N \times 2 N$ matrix, $T$, is a $\operatorname{Sp}(N)$ generator, i.e. $T^{\dagger}=-T, T^{t} \mathcal{E}+\mathcal{E} T=0$.

5. Special superconformal transformations

$$
\begin{aligned}
& \delta x^{A}=2 x \cdot b x^{A}-x^{2} b^{A}+\bar{\theta}_{i} \mathrm{~b} \theta^{j} \bar{\theta}_{j} \gamma^{A} \theta^{i}+\bar{\theta}_{i} \gamma^{A} \tilde{\mathrm{x}}_{+} \rho^{i} \\
& \delta \theta^{i}=\tilde{\mathrm{x}}_{-} \mathrm{b} \theta^{i}+i \tilde{\mathrm{x}}_{-} \rho^{i}-4\left(\bar{\rho}_{j} \theta^{i}\right) \theta^{j}
\end{aligned}
$$

\section{$3.2(N, \tilde{N}), N, \tilde{N} \neq 0$ Case}

To get the generators of $(N, \tilde{N})$ possible superconformal transformations, where both $N, \tilde{N} \neq 0$, we need to require $h^{A}$ to satisfy eq.(3.10) as well. $\left\{D_{i \alpha}, \tilde{D}_{\tilde{\jmath}}^{\beta}\right\}=0$ implies

$$
\tilde{D}_{\tilde{\jmath}}^{\beta}\left(\bar{\lambda}_{i} \gamma^{A}\right)_{\alpha}=-D_{i \alpha}\left(\overline{\tilde{\lambda}}_{\tilde{\jmath}} \tilde{\gamma}^{A}\right)^{\beta}
$$

Contracting this with $\tilde{\gamma}_{A}^{\gamma \delta}$, using eqs.(2.9, A.1a), leads to

$$
2 \tilde{D}_{\tilde{\jmath}}^{\beta} \bar{\lambda}_{i}^{[\gamma} \delta_{\alpha}^{\delta]}=D_{i \alpha} \overline{\tilde{\lambda}}_{\tilde{\jmath} \eta} \epsilon^{\eta \beta \gamma \delta}
$$

Furthermore considering the contraction with $\delta_{\beta \gamma}$, it is easy to show $D_{i \alpha} \overline{\tilde{\lambda}}_{\tilde{\jmath}}=\tilde{D}_{\tilde{\imath}}^{\alpha} \bar{\lambda}_{j}^{\beta}=0$ and so

$$
D_{i \alpha} \tilde{D}_{\tilde{\jmath}}^{\beta} h^{A}=0
$$

Consequently

$$
\begin{gathered}
D_{i \alpha} \partial_{B} h^{A}=\tilde{D}_{\tilde{\imath}}^{\alpha} \partial_{B} h^{A}=0 \\
\partial_{B} \partial_{C} h^{A}=0
\end{gathered}
$$

which imply that if $N, \tilde{N} \neq 0$ then there are no special superconformal transformations as in eq. (3.48).

The infinitesimal transformations satisfying eq.(3.1) are just

1. Supertranslations

$$
\delta x^{A}=a^{A}-i \bar{\theta}_{i} \gamma^{A} \varepsilon^{i}-i \overline{\tilde{\theta}}_{\tilde{\imath}} \tilde{\gamma}^{A} \tilde{\varepsilon}^{\tilde{\imath}} \quad \delta \theta^{i}=\varepsilon^{i} \quad \delta \tilde{\theta}^{\tilde{\imath}}=\tilde{\varepsilon}^{\tilde{\imath}}
$$


2. Dilations

$$
\delta x^{A}=\lambda x^{A} \quad \delta \theta^{i}=\frac{1}{2} \lambda \theta^{i} \quad \delta \tilde{\theta}^{\tilde{\imath}}=\frac{1}{2} \lambda \tilde{\theta}^{\tilde{i}}
$$

3. Lorentz transformations

$$
\delta x^{A}=\omega_{B}^{A} x^{B} \quad \delta \theta^{i}=\omega \theta^{i} \quad \delta \tilde{\theta}^{\tilde{i}}=\tilde{\omega} \tilde{\theta}^{\tilde{i}}
$$

4. $R$-symmetry transformations, $\operatorname{Sp}(N) \times \operatorname{Sp}(\tilde{N})$

$$
\delta x^{A}=0 \quad \delta \theta^{i}=-\theta^{j} T_{j}^{i} \quad \delta \tilde{\theta}^{\tilde{\imath}}=-\tilde{\theta}^{\tilde{\jmath}} \tilde{T}_{\tilde{\jmath}}^{\tilde{\imath}}
$$

where $T, \tilde{T}$ are $\operatorname{Sp}(N), \operatorname{Sp}(\tilde{N})$ generators respectively.

The supersymmetric interval in this case, which is invariant under supertranslations (3.53), is given by

$$
z_{12}^{\mathcal{A}}=\left(x_{12}^{A}, \theta_{12}^{i}, \tilde{\theta}_{12}^{\tilde{\imath}}\right)=-z_{21}^{\mathcal{A}}
$$

where

$$
x_{12}^{A}=x_{1}^{A}-x_{2}^{A}-i \bar{\theta}_{2 i} \gamma^{A} \theta_{1}^{i}-i \overline{\tilde{\theta}}_{2 i} \tilde{\gamma}^{A} \tilde{\theta}_{1}^{\tilde{i}} \quad \theta_{12}^{i}=\theta_{1}^{i}-\theta_{2}^{i} \quad \tilde{\theta}_{12}^{\tilde{\imath}}=\tilde{\theta}_{1}^{\tilde{i}}-\tilde{\theta}_{2}^{\tilde{i}}
$$

\subsection{Superinversion and Spatial Reflection}

In this subsection we show how superinversion may be defined as a map from $\mathbb{R}_{+}^{6 \mid 8 N}$ superspace to $\mathbb{R}_{-}^{6 \mid 8 N}$ superspace, where $\mathbb{R}_{-}^{6 \mid 8 N}$ has coordinates $\tilde{z}^{\tilde{\mathcal{A}}}=\left(y^{A}, \phi^{i}\right)$ with $\phi_{\alpha}^{i}$ having the opposite chirality to $\theta^{i \alpha}$.

For any $z^{\mathcal{A}}=\left(x^{A}, \theta^{i}\right) \in \mathbb{R}_{+}^{6 \mid 8 N}$ if we define $\mathrm{y}_{ \pm}, \phi^{i}, \bar{\phi}_{i}$ by

$$
\mathrm{y}_{ \pm}=-\tilde{\mathrm{x}}_{ \pm}^{-1} \quad \phi^{i}=-i \tilde{\mathrm{x}}_{-}^{-1} \theta^{i} \quad \bar{\phi}_{i}=i \bar{\theta}_{i} \tilde{\mathrm{x}}_{+}^{-1}
$$

then from eqs.(2.21, 3.24) these satisfy

$$
\begin{gathered}
\mathrm{y}_{+}-\mathrm{y}_{-}=\tilde{\mathrm{x}}_{-}^{-1}\left(\tilde{\mathrm{x}}_{+}-\tilde{\mathrm{x}}_{-}\right) \tilde{\mathrm{x}}_{+}^{-1}=4 i \phi^{i} \bar{\phi}_{i} \\
\phi^{i \dagger} \gamma^{0}=\phi^{j t} \overline{\mathcal{E}}_{j i}=\bar{\phi}_{i}
\end{gathered}
$$

Hence

$$
\mathrm{y}=\frac{1}{2}\left(\mathrm{y}_{+}+\mathrm{y}_{-}\right)=-\mathrm{y}^{t}=y^{A} \gamma_{A}
$$


and in consequence $\tilde{z}^{\tilde{\mathcal{A}}}=\left(y^{A}, \phi^{i}\right) \in \mathbb{R}_{-}^{6 \mid 8 N}$. We may therefore use eq.(3.59) to define superinversion $z^{\mathcal{A}} \stackrel{i}{\longrightarrow} \tilde{z}^{\tilde{\mathcal{A}}}$ as a map, $\quad \mathbb{R}_{+}^{6 \mid 8 N} \rightarrow \mathbb{R}_{-}^{6 \mid 8 N}$.

From eq.(3.59) the inverse $i^{-1}$ is easily seen to be given by

$$
\tilde{\mathrm{x}}_{ \pm}=-\mathrm{y}_{ \pm}^{-1} \quad \theta^{i}=-i \mathrm{y}_{-}^{-1} \phi^{i} \quad \bar{\theta}_{i}=i \bar{\phi}_{i} \mathrm{y}_{+}^{-1}
$$

From eq.(2.26) the infinitesimal supersymmetric intervals, $e^{A}(z), f^{A}(\tilde{z})$ for the $\mathbb{R}_{+}^{6 \mid 8 N}$ and $\mathbb{R}_{-}^{6 \mid 8 N}$ superspaces are respectively

$$
e^{A}(z)=\mathrm{d} x^{A}-i \bar{\theta}_{i} \gamma^{A} \mathrm{~d} \theta^{i} \quad f^{A}(\tilde{z})=\mathrm{d} y^{A}-i \bar{\phi}_{i} \tilde{\gamma}^{A} \mathrm{~d} \phi^{i}
$$

Under superinversion, $e^{A}$ transforms as

$$
\mathrm{f}(\tilde{z})=\tilde{\mathrm{x}}_{-}^{-1} \tilde{\mathrm{e}}(z) \tilde{\mathrm{x}}_{+}^{-1}
$$

or equivalently

$$
f^{A}(\tilde{z})=e^{B}(z) R_{B}^{A}(z ; i) \quad R_{B}^{A}(z ; i)=\frac{1}{4} \operatorname{tr}\left(\tilde{\mathrm{x}}_{-}^{-1} \tilde{\gamma}_{B} \tilde{\mathrm{x}}_{+}^{-1} \tilde{\gamma}^{A}\right)
$$

If we consider a transformation, $\tilde{z} \stackrel{i^{-1}}{\longrightarrow} z \stackrel{g}{\longrightarrow} z^{\prime} \stackrel{i}{\longrightarrow} \tilde{z}^{\prime}$, where $g$ is a $(N, 0)$ superconformal transformation, then this becomes a $(0, N)$ superconformal transformation $\tilde{z} \stackrel{\tilde{g}}{\longrightarrow} \tilde{z}^{\prime}$. To demonstrate we consider the variations $\delta \mathrm{y}_{+}, \delta \phi^{i}$ arising from the infinitesimal $(N, 0)$ superconformal transformation (3.41, 3.42a)

$$
\begin{aligned}
& \delta \mathrm{y}_{+}=\tilde{\mathrm{x}}_{+}^{-1} \delta \tilde{\mathrm{x}}_{+} \tilde{\mathrm{x}}_{+}^{-1}=\mathrm{y}_{+} \tilde{\mathrm{a}} \mathrm{y}_{+}-4 \mathrm{y}_{+} \varepsilon^{i} \bar{\phi}_{i}-\lambda \mathrm{y}_{+}+\tilde{\omega} \mathrm{y}_{+}-\mathrm{y}_{+} \omega+4 i \rho^{i} \bar{\phi}_{i}+\mathrm{b} \\
& \delta \phi^{i}=\rho^{i}-\frac{1}{2} \lambda \phi^{i}+\tilde{\omega} \phi^{i}-\phi^{j} T_{j}^{i}+\mathrm{y}_{-} \tilde{\mathrm{a}} \phi^{i}+i \mathrm{y}_{-} \varepsilon^{i}-4\left(\bar{\varepsilon}_{j} \phi^{i}\right) \phi^{j}
\end{aligned}
$$

Hence, under superinversion, the superconformal transformations are related by

$$
\left(\begin{array}{c}
a \\
b \\
\varepsilon^{i} \\
\rho^{i} \\
\lambda \\
\omega_{A B} \\
T_{i}^{j}
\end{array}\right) \quad \longrightarrow \quad\left(\begin{array}{c}
b \\
a \\
\rho^{i} \\
\varepsilon^{i} \\
-\lambda \\
\omega_{A B} \\
T_{i}^{j}
\end{array}\right)
$$

which manifestly shows a duality between $(N, 0)$ and $(0, N)$ superconformal transformations. In particular, $(N, 0)$ special superconformal transformations (3.48) can be obtained 
by $z \stackrel{i}{\longrightarrow} \tilde{z} \stackrel{(b, \rho)}{\longrightarrow} \tilde{z}^{\prime} \stackrel{i^{-1}}{\longrightarrow} z^{\prime}$ where $(b, \rho)$ is a supertranslation on $\mathbb{R}_{-}^{6 \mid 8 N}$ superspace.

We may also define a reflection map, $r_{B} B \neq 0$, from $\mathbb{R}_{+}^{6 \mid 8 N}$ superspace to $\mathbb{R}_{-}^{6 \mid 8 N}$ superspace so that $z^{\mathcal{A}} \stackrel{r_{B}}{\longrightarrow} \tilde{z}^{\tilde{\mathcal{A}}}$ by

$$
y^{A}=\left\{\begin{array}{cc}
x^{A} & A \neq B \\
-x^{A} & A=B
\end{array} \quad \text { or } \quad \mathrm{y}=\gamma^{B} \tilde{\mathrm{x}} \gamma^{B}\right.
$$

and, introducing in addition a $\operatorname{Sp}(N)$ transformation, $R$,

$$
\phi^{i}=i \gamma^{B} \theta^{j} R_{j}{ }^{i} \quad \bar{\phi}_{i}=-i\left(R^{-1}\right)_{i}{ }^{j} \bar{\theta}_{j} \gamma^{B}
$$

This gives with the definitions (3.63)

$$
\mathrm{f}(\tilde{z})=\gamma^{B} \tilde{\mathrm{e}}(z) \gamma^{B}
$$

We may now define a map $z \stackrel{i}{\longrightarrow} \tilde{z} \stackrel{r_{B}^{-1}}{\longrightarrow} z^{\prime}$ or $z \stackrel{i_{B}}{\longrightarrow} z^{\prime}$ which leads

$$
\tilde{\mathrm{x}}_{ \pm}^{\prime}=-\tilde{\gamma}^{B} \tilde{\mathrm{x}}_{ \pm}^{-1} \tilde{\gamma}^{B} \quad \theta^{i}=\tilde{\gamma}^{B} \tilde{\mathrm{x}}_{-}^{-1} \theta^{j}\left(R^{-1}\right)_{j}{ }^{i} \quad \bar{\theta}_{i}^{\prime}=R_{i}{ }^{j} \bar{\theta}_{j} \tilde{\mathrm{x}}_{+}^{-1} \tilde{\gamma}^{B}
$$

It is easy to see since $\tilde{\mathbf{x}}_{ \pm}^{\prime-1}=-\gamma^{B} \tilde{\mathrm{x}}_{ \pm} \gamma^{B}$ that

$$
\left(x^{A}, \theta^{i}\right) \stackrel{i_{B}^{2}}{\longrightarrow}\left(x^{A},-\theta^{j}\left(R^{2}\right)^{-1}{ }_{j}^{i}\right)
$$

If we choose $R$ so that

$$
R^{2}=-1
$$

then

$$
i_{B}^{2}=1
$$

\section{$3.4(N, 0)$ Superconformal Algebra}

The generator of infinitesimal $(N, 0)$ superconformal transformations, $\mathcal{L}$, is given by

$$
\mathcal{L}=h^{A} \partial_{A}+\lambda^{i \alpha} D_{i \alpha}
$$

If we write the commutator of two generators, $\mathcal{L}_{1}, \mathcal{L}_{2}$ as

$$
\left[\mathcal{L}_{2}, \mathcal{L}_{1}\right]=\mathcal{L}_{3}=h_{3}^{A} \partial_{A}+\lambda_{3}^{i \alpha} D_{i \alpha}
$$


then $h_{3}^{A}, \lambda_{3}^{i \alpha}$ are given by

$$
\begin{aligned}
& h_{3}^{A}=h_{2}^{B} \partial_{B} h_{1}^{A}-i \bar{\lambda}_{2 i} \gamma^{A} \lambda_{1}^{i}-(1 \leftrightarrow 2) \\
& \lambda_{3}^{i \alpha}=h_{2}^{A} \partial_{A} \lambda_{1}^{i \alpha}+\lambda_{2}^{j \beta} D_{j \beta} \lambda_{1}^{i \alpha}-(1 \leftrightarrow 2)
\end{aligned}
$$

and $h_{3}^{A}, \lambda_{3}^{i \alpha}$ satisfy eq.(3.9) verifying the closure of the Lie algebra.

Explicitly we getf

$$
\begin{aligned}
& a_{3}^{A}=\omega_{1 B}^{A} a_{2}^{B}+\lambda_{1} a_{2}^{A}+i \bar{\varepsilon}_{1 i} \gamma^{A} \varepsilon_{2}^{i}-(1 \leftrightarrow 2) \\
& \varepsilon_{3}^{i}=\omega_{1} \varepsilon_{2}^{i}+\frac{1}{2} \lambda_{1} \varepsilon_{2}^{i}+i \tilde{\mathrm{a}}_{2} \rho_{1}^{i}-\varepsilon_{2}^{j} T_{1 j}{ }^{i}-(1 \leftrightarrow 2) \\
& \lambda_{3}=2 a_{2} \cdot b_{1}+2 \bar{\rho}_{1 i} \varepsilon_{2}^{i}-(1 \leftrightarrow 2) \\
& \omega_{3}^{A B}=\omega_{1 C}^{A} \omega_{2}^{C B}+2\left(a_{2}^{A} b_{1}^{B}-a_{2}^{B} b_{1}^{A}\right)+2 \bar{\varepsilon}_{2 i} \gamma^{[A} \tilde{\gamma}^{B]} \rho_{1}^{i}-(1 \leftrightarrow 2) \\
& b_{3}^{A}=\omega_{1 B}^{A} b_{2}^{B}-\lambda_{1} b_{2}^{A}+i \bar{\rho}_{1 i} \tilde{\gamma}^{A} \rho_{2}^{i}-(1 \leftrightarrow 2) \\
& \rho_{3}^{i}=\tilde{\omega}_{1} \rho_{2}^{i}-\frac{1}{2} \lambda_{1} \rho_{2}^{i}+i \mathrm{~b}_{2} \varepsilon_{1}^{i}-\rho_{2}^{j} T_{1 j}{ }^{i}-(1 \leftrightarrow 2) \\
& T_{3 i}{ }^{j}=\left(T_{1} T_{2}\right)_{i}{ }^{j}+4\left(\bar{\varepsilon}_{1 i} \rho_{2}^{j}+\bar{\rho}_{1 i} \varepsilon_{2}^{j}\right)-(1 \leftrightarrow 2)
\end{aligned}
$$

Now, if we define a $(8+2 N) \times(8+2 N)$ matrix, $M$ as

$$
M=\left(\begin{array}{ccc}
\omega+\frac{1}{2} \lambda & -i \tilde{\mathrm{a}} & 2 \varepsilon^{j} \\
-i \mathrm{~b} & \tilde{\omega}-\frac{1}{2} \lambda & 2 \rho^{j} \\
2 \bar{\rho}_{i} & 2 \bar{\varepsilon}_{i} & T_{i}^{j}
\end{array}\right)
$$

then the relation above (3.78) agrees with the matrix commutator

$$
\left[M_{1}, M_{2}\right]=M_{3}
$$

This can be verified from eqs. 2.9, 2.10) using

$$
\begin{gathered}
\bar{\varepsilon}_{1 i} \gamma^{A} \varepsilon_{2}^{i} \tilde{\gamma}_{A}=2\left(\varepsilon_{1}^{i} \bar{\varepsilon}_{2 i}-\varepsilon_{2}^{i} \bar{\varepsilon}_{1 i}\right) \\
\rho_{1}^{i} \bar{\varepsilon}_{2 i}=\frac{1}{8} \bar{\varepsilon}_{2 i} \gamma^{[A} \tilde{\gamma}^{B]} \rho_{1}^{i} \gamma_{[A} \tilde{\gamma}_{B]}-\frac{1}{4} \bar{\varepsilon}_{2 i} \rho_{1}^{i} 1
\end{gathered}
$$

\footnotetext{
${ }^{4}$ From eq. $(3.78)$ we can read off the six-dimensional $(N, 0)$ superconformal algebra. The explicit form of it is given in Appendix C.
} 
In general, $M$ can be defined as a $(8,2 N)$ supermatrix subject to the two conditions?

$$
\begin{aligned}
& B M B^{-1}=-M^{\dagger} \\
& C M C^{-1}=-M^{t}
\end{aligned}
$$

where

$$
B=B^{-1}=\left(\begin{array}{ccc}
0 & \tilde{\gamma}^{0} & 0 \\
\gamma^{0} & 0 & 0 \\
0 & 0 & -1
\end{array}\right), \quad C=\left(\begin{array}{ccc}
0 & 1 & 0 \\
1 & 0 & 0 \\
0 & 0 & -\mathcal{E}
\end{array}\right)
$$

The $8 \times 8$ matrix appearing in $M$

$$
\left(\begin{array}{cc}
\omega+\frac{1}{2} \lambda & -i \tilde{\mathrm{a}} \\
-i \mathrm{~b} & \tilde{\omega}-\frac{1}{2} \lambda
\end{array}\right)
$$

corresponds to a generator of $\mathrm{O}(2,6)$ as demonstrated in Appendix D. Thus, the $(N, 0)$ superconformal group in six-dimensions may be identified with the matrix group generated by matrices of the form $M$, which is $\operatorname{OSp}(2,6 \mid N) \equiv \mathrm{G}_{s}$ having dimensions $(28+N(2 N+1) \mid 16 N)$.

\section{Coset Realization of Transformations}

To obtain an explicit formula for the finite superconformal transformations, we first identify the superspace, $\mathbb{R}_{+}^{6 / 8 N}$, as a coset, $\mathrm{G}_{s} / \mathrm{G}_{0}$, where $\mathrm{G}_{0} \subset \mathrm{G}_{s}$ is the subgroup generated by matrices $M_{0}$ of the form (3.79) with $a^{A}=0, \varepsilon^{i}=0$ and depending on parameters $b^{A}, \rho^{i}, \lambda, \omega_{A B}, T_{i}{ }^{j}$.

The group of supertranslations, $\mathrm{G}_{T}$, is parameterized by coordinates, $z^{\mathcal{A}} \in \mathbb{R}_{+}^{6 \mid 8 N}$, and is defined in terms of supermatrices]

$$
G_{T}(z)=\exp \left(\begin{array}{ccc}
0 & -i \tilde{\mathrm{x}} & 2 \theta^{j} \\
0 & 0 & 0 \\
0 & 2 \bar{\theta}_{i} & 0
\end{array}\right)=\left(\begin{array}{ccc}
1 & -i \tilde{\mathrm{x}}_{+} & 2 \theta^{j} \\
0 & 1 & 0 \\
0 & 2 \bar{\theta}_{i} & \delta_{i}{ }^{j}
\end{array}\right)
$$

where $\tilde{\mathrm{x}}_{+}$is given in eq.(3.23).

We may write

$$
G_{T}\left(z_{2}\right)^{-1} G_{T}\left(z_{1}\right)=G_{T}\left(z_{12}\right) \quad G_{T}(z)^{-1}=G_{T}(-z)
$$

${ }^{5}$ We define the transpose of a supermatrix as $\left(\begin{array}{ll}a & b \\ c & d\end{array}\right)^{t}=\left(\begin{array}{cc}a^{t} & c^{t} \\ -b^{t} & d^{t}\end{array}\right)$.

${ }^{6}$ The subscript, $T$, denotes supertranslations. 
where

$$
\begin{gathered}
z_{12}^{\mathcal{A}}=\left(x_{12}^{A}, \theta_{12}^{i}\right)=-z_{21}^{\mathcal{A}} \\
x_{12}^{A}=x_{1}^{A}-x_{2}^{A}-i \bar{\theta}_{2 i} \gamma^{A} \theta_{1}^{i} \quad \theta_{12}^{i}=\theta_{1}^{i}-\theta_{2}^{i}
\end{gathered}
$$

which defines supersymmetric interval for $\mathbb{R}_{+}^{6 \mid 8 N}$ superspace, as in eq.(3.58).

In general an element of $\mathrm{G}_{s}$ can be uniquely decomposed as $G_{T} G_{0}$. Thus for any element $G(g) \in \mathrm{G}_{s}$ we may define a superconformal transformation, $z \stackrel{g}{\longrightarrow} z^{\prime}$, and an associated element $G_{0}(z ; g) \in \mathrm{G}_{0}$ by

$$
G(g)^{-1} G_{T}(z) G_{0}(z ; g)=G_{T}\left(z^{\prime}\right)
$$

If $G(g) \in \mathrm{G}_{T}$ then clearly $G_{0}(z ; g)=1$.

Infinitesimally eq.(4.4) becomes

$$
\delta G_{T}(z)=M G_{T}(z)-G_{T}(z) \hat{M}_{0}(z)
$$

If $M$ is given by eq.(3.79) then letting $\delta G_{T}(z)=\mathcal{L} G_{T}(z)$ we may verify that $\mathcal{L}$ is identical with the form given by eq. (3.75) with eqs. 3.40, 3.41) and further $\hat{M}_{0}(z)$, the generator of $\mathrm{G}_{0}$, has the form

$$
\hat{M}_{0}(z)=\left(\begin{array}{ccc}
\hat{\omega}(z)+\frac{1}{2} \hat{\lambda}(z) & 0 & 0 \\
-i \mathrm{~b} & \hat{\hat{\omega}}(z)-\frac{1}{2} \hat{\lambda}(z) & 2 \hat{\rho}^{j}(z) \\
2 \overline{\hat{\rho}}_{i}(z) & 0 & \hat{T}_{i}{ }^{j}(z)
\end{array}\right)
$$

where the elements of $\hat{M}_{0}(z)$ depending on $z$ are given by

$$
\begin{aligned}
& \hat{\omega}(z)=\omega-4 \theta^{i} \bar{\rho}_{i}+\tilde{\mathrm{x}}_{-} \mathrm{b}+\frac{1}{4} \operatorname{tr}\left(4 \theta^{i} \bar{\rho}_{i}-\tilde{\mathrm{x}}_{-} \mathrm{b}\right) 1 \\
& \hat{\hat{\omega}}(z)=\tilde{\omega}+4 \rho^{i} \bar{\theta}_{i}-\mathrm{b} \tilde{\mathrm{x}}_{+}-\frac{1}{4} \operatorname{tr}\left(4 \rho^{i} \bar{\theta}_{i}-\mathrm{b} \tilde{\mathrm{x}}_{+}\right) 1=-\hat{\omega}(z)^{t} \\
& \hat{\lambda}(z)=\lambda+2 b \cdot x+2 \bar{\theta}_{i} \rho^{i}=\frac{1}{6} \partial_{A} h^{A}(z) \\
& \hat{T}_{i}^{j}(z)=T_{i}^{j}+4 i \bar{\theta}_{i} \mathrm{~b} \theta^{j}+4\left(\bar{\rho}_{i} \theta^{j}-\bar{\theta}_{i} \rho^{j}\right) \\
& \hat{\rho}^{j}(z)=\rho^{j}-i \mathrm{~b} \theta^{j}=-i \frac{1}{6} \gamma^{A} \partial_{A} \lambda^{j}(z)
\end{aligned}
$$

$\hat{\omega}(z), \tilde{\hat{\omega}}(z)$ can be also written as $\hat{\omega}(z)=\frac{1}{4} \hat{\omega}_{A B}(z) \tilde{\gamma}^{A} \gamma^{B}, \tilde{\hat{\omega}}(z)=\frac{1}{4} \hat{\omega}_{A B}(z) \gamma^{A} \tilde{\gamma}^{B}$ where

$$
\hat{\omega}_{A B}(z)=\omega_{A B}+4 x_{[A} b_{B]}+\bar{\theta}_{i} \gamma_{[A} \tilde{\gamma}_{B]}\left(2 \rho^{i}-i \mathrm{~b} \theta^{i}\right)=-\partial_{[A} h_{B]}(z)
$$


The definitions (4.7) may be summarized by

$$
D_{i \alpha} \lambda^{j \beta}(z)=\delta_{i}{ }^{j}\left(\frac{1}{2} \hat{\lambda}(z) \delta_{\alpha}^{\beta}-\tilde{\hat{\omega}}_{\alpha}^{\beta}(z)\right)-\hat{T}_{i}{ }^{j}(z) \delta_{\alpha}{ }^{\beta}
$$

and they give

$$
\left[D_{i \alpha}, \mathcal{L}\right]=\frac{1}{2} \hat{\lambda}(z) D_{i \alpha}-\tilde{\hat{\omega}}_{\alpha}^{\beta}(z) D_{i \beta}-\hat{T}_{i}^{j}(z) D_{j \alpha}
$$

For later use we note

$$
\begin{aligned}
& D_{i \alpha} \tilde{\hat{\omega}}_{\beta}^{\gamma}(z)=4 \delta_{\alpha}^{\gamma} \overline{\hat{\rho}}_{i \beta}(z)-\delta_{\beta}^{\gamma} \overline{\hat{\rho}}_{i \alpha}(z) \\
& D_{i \alpha} \hat{\lambda}(z)=-2 \overline{\hat{\rho}}_{i \alpha}(z) \\
& D_{i \alpha} \hat{T}_{j}^{k}(z)=-4 \delta_{i}{ }^{k} \overline{\hat{\rho}}_{j \alpha}(z)-4 \overline{\mathcal{E}}_{i j} \hat{\rho}_{\alpha}^{k}(z)
\end{aligned}
$$

The above analysis can be simplified by reducing $G_{0}(z ; g)$. To achieve this we let

$$
Z_{0}=\left(\begin{array}{ll}
0 & 0 \\
1 & 0 \\
0 & 1
\end{array}\right)
$$

and then

$$
M_{0} Z_{0}=Z_{0} H_{0} \quad H_{0}=\left(\begin{array}{cc}
\tilde{\omega}-\frac{1}{2} \lambda & 2 \rho^{j} \\
0 & T_{i}^{j}
\end{array}\right)
$$

Now if we define

$$
Z(z) \equiv G_{T}(z) Z_{0}=\left(\begin{array}{cc}
-i \tilde{\mathrm{x}}_{+} & 2 \theta^{j} \\
1 & 0 \\
2 \bar{\theta}_{i} & \delta_{i}{ }^{j}
\end{array}\right)
$$

then $Z(z)$ transforms under infinitesimal superconformal transformations as

$$
\delta Z(z)=\mathcal{L} Z(z)=M Z(z)-Z(z) H(z)
$$

where $H(z)$ is given by

$$
\hat{M}_{0}(z) Z_{0}=Z_{0} H(z) \quad H(z)=\left(\begin{array}{cc}
\tilde{\hat{\omega}}(z)-\frac{1}{2} \hat{\lambda}(z) & 2 \hat{\rho}^{j}(z) \\
0 & \hat{T}_{i}^{j}(z)
\end{array}\right)
$$

From eqs. 3.76, 3.80) we have

$$
H_{3}(z)=\mathcal{L}_{2} H_{1}(z)-\mathcal{L}_{1} H_{2}(z)+\left[H_{1}(z), H_{2}(z)\right]
$$


which gives separate equations for $\tilde{\hat{\omega}}, \hat{\lambda}, \hat{\rho}^{i}$ and $\hat{T}_{i}{ }^{j}$, thus $\hat{\lambda}_{3}=\mathcal{L}_{2} \hat{\lambda}_{1}-\mathcal{L}_{1} \hat{\lambda}_{2}$, etc.

As a conjugate of $Z(z)$ we define $\bar{Z}(z)$ by

$$
\bar{Z}(z)=\left(\begin{array}{cc}
\tilde{\gamma}^{0} & 0 \\
0 & -1
\end{array}\right) Z(z)^{\dagger} B=\left(\begin{array}{cc}
1 & 0 \\
0 & -\overline{\mathcal{E}}
\end{array}\right) Z(z)^{t} C=\left(\begin{array}{ccc}
1 & i \tilde{\mathrm{x}}_{-} & -2 \theta^{j} \\
0 & -2 \bar{\theta}_{i} & \delta_{i}^{j}
\end{array}\right)
$$

This satisfies

$$
\bar{Z}(z)=\bar{Z}(0) G_{T}(z)^{-1}
$$

and corresponding to eq.(4.15) we have

$$
\delta \bar{Z}(z)=\mathcal{L} \bar{Z}(z)=\bar{H}(z) \bar{Z}(z)-\bar{Z}(z) M
$$

where

$$
\bar{H}(z)=\left(\begin{array}{cc}
\hat{\omega}(z)+\frac{1}{2} \hat{\lambda}(z) & 0 \\
2 \overline{\hat{\rho}}_{i}(z) & \hat{T}_{i}^{j}(z)
\end{array}\right)
$$

\subsection{Functions of Two Points}

To consider functions of two points, $z_{1}, z_{2}$, which transform covariantly under superconformal transformations, we first define $F(z)$ for $z \in \mathbb{R}_{+}^{6 \mid 8 N}$ by

$$
F(z)=\left(\begin{array}{cc}
-i \tilde{\mathrm{x}}_{+} & 2 \theta^{j} \\
2 \bar{\theta}_{i} & \delta_{i}^{j}
\end{array}\right)
$$

This satisfies the conditions

$$
\begin{aligned}
F(-z) & =\left(\begin{array}{cc}
\tilde{\gamma}^{0} & 0 \\
0 & -1
\end{array}\right) F(z)^{\dagger}\left(\begin{array}{cc}
\tilde{\gamma}^{0} & 0 \\
0 & -1
\end{array}\right)=\left(\begin{array}{cc}
1 & 0 \\
0 & -\overline{\mathcal{E}}
\end{array}\right) F(z)^{t}\left(\begin{array}{cc}
1 & 0 \\
0 & -\mathcal{E}
\end{array}\right) \\
& =\left(\begin{array}{cc}
i \tilde{\mathrm{x}}_{-} & -2 \theta^{j} \\
-2 \bar{\theta}_{i} & \delta_{i}^{j}
\end{array}\right)
\end{aligned}
$$

and the superdeterminant of $F(z)$ is given by

$$
\begin{aligned}
\operatorname{sdet} F(z) & =\operatorname{det}\left(-i \tilde{\mathrm{x}}_{+}-4 \theta^{i} \bar{\theta}_{i}\right)=\operatorname{det}\left(-i \tilde{\mathrm{x}}_{-}\right) \\
& =\operatorname{det} \tilde{\mathrm{x}}_{+}=\operatorname{det} \tilde{\mathrm{x}}_{-}
\end{aligned}
$$

If we consider

$$
\left(\begin{array}{cc}
1 & 0 \\
-2 i \bar{\theta}_{i} \tilde{\mathrm{x}}_{+}^{-1} & 1
\end{array}\right) F(z)\left(\begin{array}{cc}
1 & -2 i \tilde{\mathrm{x}}_{+}^{-1} \theta^{j} \\
0 & 1
\end{array}\right)=\left(\begin{array}{cc}
-i \tilde{\mathrm{x}}_{+} & 0 \\
0 & V_{i}^{j}(-z)
\end{array}\right)
$$


then this defines $V_{i}^{j}(z)$ as

$$
V_{i}^{j}(z)=\delta_{i}{ }^{j}+4 i \bar{\theta}_{i} \tilde{\mathrm{x}}_{-}^{-1} \theta^{j}
$$

From eqs. (4.24, 4.25) it is evident that

$$
\operatorname{det} V(z)=1
$$

and from eq.(3.24) $V_{i}^{j}(z)$ also satisfies eq.(4.52) and hence $V_{i}^{j}(z) \in \operatorname{Sp}(N)$. Furthermore we have

$$
V_{i}{ }^{j}(-z)=V^{-1}{ }_{i}{ }^{j}(z)=V^{\dagger}{ }_{i}{ }^{j}(z)=\delta_{i}{ }^{j}-4 i \bar{\theta}_{i} \tilde{\mathrm{x}}_{+}^{-1} \theta^{j}
$$

We may also show from eq.(4.25)

$$
F(z)^{-1}=\left(\begin{array}{cc}
i \tilde{\mathrm{x}}_{-}^{-1} & -2 i \tilde{\mathrm{x}}_{-}^{-1} \theta^{j} \\
-2 i \bar{\theta}_{i} \tilde{\mathrm{x}}_{-}^{-1} & V_{i}{ }^{j}(z)
\end{array}\right)
$$

Using the definition of $F(z)$ (4.22), we may now write from eqs.(4.14, 4.18)

$$
\bar{Z}\left(z_{2}\right) Z\left(z_{1}\right)=F\left(z_{12}\right)=\left(\begin{array}{cc}
i \mathcal{X}_{21} & -2 \theta_{21}^{j} \\
-2 \bar{\theta}_{21 i} & \delta_{i}{ }^{j}
\end{array}\right)
$$

where from eq.(3.23)

$$
\begin{aligned}
\mathcal{X}_{21} & =\tilde{\mathrm{x}}_{2-}-\tilde{\mathrm{x}}_{1+}+4 i \theta_{2}^{i} \bar{\theta}_{1 i} \\
& =\tilde{\mathrm{x}}_{21}-2 i \theta_{21}^{i} \bar{\theta}_{21 i}=\left(\tilde{\mathrm{x}}_{21}\right)_{-}=-\left(\tilde{\mathrm{x}}_{12}\right)_{+}
\end{aligned}
$$

From eq.(3.24) we also have

$$
\mathcal{X}_{21}^{t}=\mathcal{X}_{12} \quad \mathcal{X}_{21}^{\dagger}=-\gamma^{0} \mathcal{X}_{12} \gamma^{0}
$$

where

$$
\mathcal{X}_{12}=\tilde{\mathrm{x}}_{12}-2 i \theta_{12}^{i} \bar{\theta}_{12 i}=-\mathcal{X}_{21}-4 i \theta_{21}^{i} \bar{\theta}_{21 i}
$$

Infinitesimally, from eqs.(4.15, 4.20), $F\left(z_{12}\right)$ transforms as

$$
\delta F\left(z_{12}\right)=\bar{H}\left(z_{2}\right) F\left(z_{12}\right)-F\left(z_{12}\right) H\left(z_{1}\right)
$$

For consistency with the form (4.30) it is necessary that

$$
\hat{T}_{i}{ }^{j}\left(z_{2}\right)-\hat{T}_{i}{ }^{j}\left(z_{1}\right)-4 \overline{\hat{\rho}}_{i}\left(z_{2}\right) \theta_{21}^{j}+4 \bar{\theta}_{21 i} \hat{\rho}^{j}\left(z_{1}\right)=0
$$


In particular, we have from eq.(4.34)

$$
\delta \mathcal{X}_{21}=\hat{\omega}\left(z_{2}\right) \mathcal{X}_{21}-\mathcal{X}_{21} \tilde{\hat{\omega}}\left(z_{1}\right)+\frac{1}{2}\left(\hat{\lambda}\left(z_{1}\right)+\hat{\lambda}\left(z_{2}\right)\right) \mathcal{X}_{21}
$$

and hence

$$
\operatorname{sdet} F\left(z_{12}\right)=\operatorname{det} \mathcal{X}_{12}=\operatorname{det} \mathcal{X}_{21}
$$

which is a scalar depending on $z_{1}, z_{2}$ transforming homogeneously

$$
\delta \operatorname{det} \mathcal{X}_{12}=2\left(\hat{\lambda}\left(z_{1}\right)+\hat{\lambda}\left(z_{2}\right)\right) \operatorname{det} \mathcal{X}_{12}
$$

From eq.(4.29) we get

$$
F\left(z_{12}\right)^{-1}=\left(\begin{array}{cc}
i \mathcal{X}_{12}^{-1} & -2 i \mathcal{X}_{12}^{-1} \theta_{12}^{j} \\
-2 i \bar{\theta}_{12 i} \mathcal{X}_{12}^{-1} & V_{i}{ }^{j}\left(z_{12}\right)
\end{array}\right)
$$

From eq.(4.34) $F\left(z_{12}\right)^{-1}$ transforms as

$$
\delta F\left(z_{12}\right)^{-1}=H\left(z_{1}\right) F\left(z_{12}\right)^{-1}-F\left(z_{12}\right)^{-1} \bar{H}\left(z_{2}\right)
$$

Consistency with the form (4.39) requires

$$
\tilde{\hat{\omega}}\left(z_{1}\right)-\frac{1}{2} \hat{\lambda}\left(z_{1}\right)-4 \hat{\rho}^{i}\left(z_{1}\right) \bar{\theta}_{12 i}+\mathrm{b} \mathcal{X}_{12}=\tilde{\hat{\omega}}\left(z_{2}\right)-\frac{1}{2} \hat{\lambda}\left(z_{2}\right)
$$

$V_{i}^{j}\left(z_{21}\right)$ transforms infinitesimally as

$$
\delta V\left(z_{21}\right)=\hat{T}\left(z_{2}\right) V\left(z_{21}\right)-V\left(z_{21}\right) \hat{T}\left(z_{1}\right)
$$

\subsection{Finite Transformations}

Finite superconformal transformations are obtained by exponentiation of infinitesimal transformations. To obtain a superconformal transformation $z \stackrel{g}{\longrightarrow} z^{\prime}$, we therefore solve the differential equation

$$
\frac{\mathrm{d}}{\mathrm{d} s} z_{s}^{\mathcal{A}}=\mathcal{L}^{\mathcal{A}}\left(z_{s}\right) \quad z_{0}=z \quad z_{1}=z^{\prime}
$$

where, with $\mathcal{L}$ given in eq. (3.75), $\mathcal{L}^{\mathcal{A}}(z)$ is defined by

$$
\mathcal{L}=\mathcal{L}^{\mathcal{A}}(z) \partial_{\mathcal{A}}
$$

From eq.(4.15) we get

$$
\frac{\mathrm{d}}{\mathrm{d} s} Z\left(z_{s}\right)=M Z\left(z_{s}\right)-Z\left(z_{s}\right) H\left(z_{s}\right)
$$


which integrates to

$$
Z\left(z_{s}\right)=e^{s M} Z(z) K(z, s)
$$

where $K(z, s)$ satisfies

$$
\frac{\mathrm{d}}{\mathrm{d} s} K(z, s)=-K(z, s) H\left(z_{s}\right) \quad K(z, 0)=\left(\begin{array}{cc}
1 & 0 \\
0 & 1
\end{array}\right)
$$

Hence for $s=1$ with $K(z, 1) \equiv K(z ; g)$ the superconformal transformation, $z \stackrel{g}{\longrightarrow} z^{\prime}$, from eq.(4.46) becomes

$$
Z\left(z^{\prime}\right)=G(g)^{-1} Z(z) K(z ; g) \quad G(g)^{-1}=e^{M}
$$

$K(z ; g)$ is a representation of the superconformal group, since under the successive superconformal transformations, $z \stackrel{g}{\longrightarrow} z^{\prime} \stackrel{g^{\prime}}{\longrightarrow} z^{\prime \prime}$ giving $z \stackrel{g^{\prime \prime}}{\longrightarrow} z^{\prime \prime}$, we have

$$
K(z ; g) K\left(z^{\prime} ; g^{\prime}\right)=K\left(z ; g^{\prime \prime}\right)
$$

In general $K(z ; g)$ is of the form

$$
K(z ; g)=\left(\begin{array}{cc}
L(z ; g) & 2 \Sigma^{j}(z ; g) \\
0 & U_{i}^{j}(z ; g)
\end{array}\right)
$$

Since $\frac{\mathrm{d}}{\mathrm{ds}} U(z, s)=-U(z, s) \hat{T}\left(z_{s}\right)$ and $\hat{T}(z)$ satisfies the conditions

$$
\hat{T}^{t} \mathcal{E}+\mathcal{E} \hat{T}=0 \quad \hat{T}^{\dagger}=-\hat{T} \quad \operatorname{tr}(\hat{T})=0
$$

$U \in \operatorname{Sp}(N)$, i.e.

$$
U^{t} \mathcal{E} U=\mathcal{E} \quad U^{\dagger}=U^{-1} \quad \operatorname{det} U=1
$$

Similarly $L$ satisfies

$$
L^{\dagger}=\gamma^{0} L^{t} \tilde{\gamma}^{0}
$$

$G_{0}(z ; g)$ in eq.(4.4) is related to $K(z ; g)$ from eq. (4.48) by

$$
G_{0}(z ; g) Z_{0}=Z_{0} K(z ; g)
$$

From eq.(4.48) $\bar{Z}$ transforms as

$$
\bar{Z}\left(z^{\prime}\right)=\bar{K}(z ; g) \bar{Z}(z) G(g)
$$


where

$$
\begin{aligned}
& \bar{K}(z ; g)=\left(\begin{array}{cc}
\tilde{\gamma}^{0} & 0 \\
0 & -1
\end{array}\right) K(z ; g)^{\dagger}\left(\begin{array}{cc}
\gamma^{0} & 0 \\
0 & -1
\end{array}\right)=\left(\begin{array}{cc}
1 & 0 \\
0 & -\overline{\mathcal{E}}
\end{array}\right) K(z ; g)^{t}\left(\begin{array}{cc}
1 & 0 \\
0 & -\mathcal{E}
\end{array}\right) \\
& =\left(\begin{array}{cc}
L(z ; g)^{t} & 0 \\
-2 \bar{\Sigma}(z ; g) & U^{-1}(z ; g)
\end{array}\right)
\end{aligned}
$$

From eqs.4.48, 4.55) $F\left(z_{12}\right)$ transforms as

$$
F\left(z_{12}^{\prime}\right)=\bar{K}\left(z_{2} ; g\right) F\left(z_{12}\right) K\left(z_{1} ; g\right)
$$

In particular, with eqs. 4.50, 4.56), this gives the transformation rule

$$
\begin{gathered}
\mathcal{X}_{21}^{\prime}=L\left(z_{2} ; g\right)^{t} \mathcal{X}_{21} L\left(z_{1} ; g\right) \\
\operatorname{det} \mathcal{X}_{12}^{\prime}=\Omega^{2}\left(z_{1} ; g\right) \Omega^{2}\left(z_{2} ; g\right) \operatorname{det} \mathcal{X}_{12}
\end{gathered}
$$

where $\Omega(z ; g)$ is given by

$$
\Omega(z ; g)^{2}=\operatorname{det} L(z ; g)=\operatorname{sdet} K(z ; g)
$$

If we write

$$
\hat{L}(z ; g)=L(z ; g) / \Omega(z ; g)^{1 / 2}
$$

then $\hat{L} \in \mathrm{G}_{L}$, the group of $4 \times 4$ matrices satisfying the reality condition eq.(4.53) and $\operatorname{det} \hat{L}=1$.

Corresponding to eq.(4.42) $V_{i}^{j}\left(z_{21}\right)$ transforms as

$$
V\left(z_{21}^{\prime}\right)=U^{-1}\left(z_{2} ; g\right) V\left(z_{21}\right) U\left(z_{1} ; g\right)
$$

\subsection{Transformation of Vectors}

If

$$
\gamma^{B} R_{B}^{A}(z ; g)=L(z ; g) \gamma^{A} L(z ; g)^{t}
$$

then $R_{A}^{B}(z ; g)$ is identical to the definition (3.2), since infinitesimally

$$
\hat{\lambda}(z) \gamma^{A}-\tilde{\hat{\omega}}(z) \gamma^{A}-\gamma^{A} \tilde{\hat{\omega}}(z)^{t}=\hat{\lambda}(z) \gamma^{A}-\gamma^{B} \hat{\omega}(z)_{B}^{A}=\gamma^{B} \partial_{B} h^{A}(z)
$$

as a consequence of eq.(4.7), which agrees with eq.(3.13). Furthermore eq.(4.63) shows that the definition (3.3) of $\Omega(z ; g)$ coincides with eq.(4.60).

From eq. (4.63)

$$
\gamma^{B} \hat{R}_{B}^{A}(z ; g)=\hat{L}(z ; g) \gamma^{A} \hat{L}(z ; g)^{t}
$$


where $\hat{R}=R / \Omega \in \mathrm{SO}(1,5) \simeq \mathrm{G}_{L} / \mathbb{Z}_{2}$.

The matrix, $\mathcal{R}_{\mathcal{A}}{ }^{\mathcal{B}}(z ; g)$, given in eq. (3.5) for the $(N, 0)$ case becomes

$$
\mathcal{R}_{\mathcal{A}}{ }^{\mathcal{B}}(z ; g)=\left(\begin{array}{cl}
R_{A}{ }^{B}(z ; g) & i\left(\Sigma^{j}(z ; g) \tilde{\gamma}_{A} L(z ; g)\right)^{\beta} \\
0 & L_{\alpha}^{\beta}(z ; g) U_{i}{ }^{j}(z ; g)
\end{array}\right)
$$

and so $D_{\mathcal{A}}$ transforms under the superconformal transformations as

$$
D_{\mathcal{A}}=\mathcal{R}_{\mathcal{A}}{ }^{\mathcal{B}}(z ; g) D_{\mathcal{B}}^{\prime}
$$

From eqs. 4.58, 4.63) $\operatorname{tr}\left(\gamma^{A} \mathcal{X}_{12} \gamma^{B} \mathcal{X}_{21}\right)$ transforms covariantly as

$$
\operatorname{tr}\left(\gamma^{A} \mathcal{X}_{12}^{\prime} \gamma^{B} \mathcal{X}_{21}^{\prime}\right)=\operatorname{tr}\left(\gamma^{C} \mathcal{X}_{12} \gamma^{D} \mathcal{X}_{21}\right) R_{C}{ }^{A}\left(z_{1} ; g\right) R_{D}{ }^{B}\left(z_{2} ; g\right)
$$

\subsection{Relation to Superinversion}

If we define

$$
\begin{aligned}
& I(z)=\left(\begin{array}{cc}
i \tilde{\mathrm{x}}_{+}^{-1} & -2 i \tilde{\mathrm{x}}_{-}^{-1} \theta^{j} \\
0 & V_{i}^{j}(z)
\end{array}\right) \\
& \bar{I}(z)=\left(\begin{array}{cc}
-i \tilde{\mathrm{x}}_{-}^{-1} & 0 \\
-2 i \bar{\theta}_{i} \tilde{\mathrm{x}}_{+}^{-1} & V^{-1}{ }_{i}{ }^{j}(z)
\end{array}\right)=\left(\begin{array}{cc}
\gamma^{0} & 0 \\
0 & -1
\end{array}\right) I(z)^{\dagger}\left(\begin{array}{cc}
\gamma^{0} & 0 \\
0 & -1
\end{array}\right)
\end{aligned}
$$

where $V_{i}^{j}(z)$ is given by eq. (4.26), then using $\theta^{i} V_{i}^{j}(z)=\tilde{\mathrm{x}}_{+} \tilde{\mathrm{x}}_{-}^{-1} \theta^{j}$ we get

$$
\begin{aligned}
& \tilde{Z}(\tilde{z}) \equiv Z(z) I(z)=\left(\begin{array}{cc}
1 & 0 \\
-i y_{+} & 2 \phi^{j} \\
2 \bar{\phi}_{i} & \delta_{i}{ }^{j}
\end{array}\right) \\
& \overline{\tilde{Z}}(\tilde{z}) \equiv \bar{I}(z) \bar{Z}(z)=\left(\begin{array}{ccc}
i y_{-} & 1 & -2 \phi^{j} \\
-2 \bar{\phi}_{i} & 0 & \delta_{i}{ }^{j}
\end{array}\right)
\end{aligned}
$$

where $\tilde{z}^{\tilde{\mathcal{A}}}=\left(y^{A}, \phi^{i}\right) \in \mathbb{R}_{-}^{6 \mid 8 N}$ is defined in eq. (3.59).

We may also write

$$
G_{T}(z) G_{I}(z)=G_{\tilde{T}}(\tilde{z})=\left(\begin{array}{ccc}
1 & 0 & 0 \\
-i \bar{y}_{+} & 1 & 2 \phi^{j} \\
2 \bar{\phi}_{i} & 0 & \delta_{i}{ }^{j}
\end{array}\right)
$$


where

$$
G_{I}(z)=\left(\begin{array}{ccc}
0 & i \tilde{\mathrm{x}}_{-} & 0 \\
i \tilde{\mathrm{x}}_{+}^{-1} & 1 & -2 i \tilde{\mathrm{x}}_{-}^{-1} \theta^{j} \\
0 & -2 \bar{\theta}_{i} & V_{i}^{j}(z)
\end{array}\right)
$$

Note

$$
\tilde{Z}(\tilde{z})=G_{\tilde{T}}(\tilde{z}) \tilde{Z}(0) \quad \overline{\tilde{Z}}(\tilde{z})=\overline{\tilde{Z}}(0) G_{\tilde{T}}(\tilde{z})^{-1}
$$

and

$$
G_{I}(z) \tilde{Z}(0)=Z(0) I(z)
$$

$G_{\tilde{T}}(\tilde{z})$ forms the group of supertranslations acting on $\tilde{z} \in \mathbb{R}_{-}^{6 \mid 8 N}$.

$\tilde{Z}(\tilde{z}), \overline{\tilde{Z}}(\tilde{z})$ transform under the superconformal transformation, $\tilde{z} \stackrel{i^{-1}}{\longrightarrow} z \stackrel{g}{\longrightarrow} z^{\prime} \stackrel{i}{\longrightarrow} \tilde{z}^{\prime}$, from eqs. $4.48,4.55)$

$$
\begin{aligned}
& \tilde{Z}\left(\tilde{z}^{\prime}\right)=G(g)^{-1} \tilde{Z}(\tilde{z}) \tilde{K}(\tilde{z} ; g) \\
& \overline{\tilde{Z}}\left(\tilde{z}^{\prime}\right)=\overline{\tilde{K}}(\tilde{z} ; g) \overline{\tilde{Z}}(\tilde{z}) G(g)
\end{aligned}
$$

where

$$
\begin{aligned}
& \tilde{K}(\tilde{z} ; g)=I(z)^{-1} K(z ; g) I\left(z^{\prime}\right) \\
& \overline{\tilde{K}}(\tilde{z} ; g)=\bar{I}\left(z^{\prime}\right) \bar{K}(z ; g) \bar{I}(z)^{-1}=\left(\begin{array}{cc}
\gamma^{0} & 0 \\
0 & -1
\end{array}\right) \tilde{K}(\tilde{z} ; g)^{\dagger}\left(\begin{array}{cc}
\tilde{\gamma}^{0} & 0 \\
0 & -1
\end{array}\right)
\end{aligned}
$$

With $z_{21} \stackrel{i}{\longrightarrow} \widetilde{z_{21}}$ we may write from eq. 4.70

$$
\tilde{Z}\left(\widetilde{z_{21}}\right)=\left(\begin{array}{cc}
1 & 0 \\
-i \mathcal{X}_{12}^{-1} & -2 i \mathcal{X}_{21}^{-1} \theta_{21}^{j} \\
-2 i \bar{\theta}_{21 i} \mathcal{X}_{12}^{-1} & \delta_{i}{ }^{j}
\end{array}\right) \quad \quad \overline{\tilde{Z}}\left(\widetilde{z_{21}}\right)=\left(\begin{array}{ccc}
-i \mathcal{X}_{21}^{-1} & 1 & 2 i \mathcal{X}_{21}^{-1} \theta_{21}^{j} \\
2 i \bar{\theta}_{21 i} \mathcal{X}_{12}^{-1} & 0 & \delta_{i}{ }^{j}
\end{array}\right)
$$

which transform, using eqs.(4.35, 4.41), according to

$$
\begin{aligned}
& \delta \tilde{Z}\left(\widetilde{z_{21}}\right)=\hat{M}_{0}\left(z_{1}\right) \tilde{Z}\left(\widetilde{z_{21}}\right)-\tilde{Z}\left(\widetilde{z_{21}}\right)\left(\begin{array}{cc}
\hat{\omega}\left(z_{1}\right)+\frac{1}{2} \hat{\lambda}\left(z_{1}\right) & 0 \\
0 & \hat{T}\left(z_{1}\right)
\end{array}\right) \\
& \delta \overline{\tilde{Z}}\left(\widetilde{z_{21}}\right)=\left(\begin{array}{cc}
\tilde{\hat{\omega}}\left(z_{1}\right)-\frac{1}{2} \hat{\lambda}\left(z_{1}\right) & 0 \\
0 & \hat{T}\left(z_{1}\right)
\end{array}\right) \overline{\tilde{Z}}\left(\widetilde{z_{21}}\right)-\overline{\tilde{Z}}\left(\widetilde{z_{21}}\right) \hat{M}_{0}\left(z_{1}\right)
\end{aligned}
$$

Taking

$$
G\left(i_{B}\right)^{-1}=\left(\begin{array}{ccc}
0 & i \tilde{\gamma}^{B} & 0 \\
-i \gamma^{B} & 0 & 0 \\
0 & 0 & R_{i}^{j}
\end{array}\right)=e^{\frac{\pi}{2} M_{B}} \quad M_{B}=\left(\begin{array}{ccc}
0 & i \tilde{\gamma}^{B} & 0 \\
-i \gamma^{B} & 0 & 0 \\
0 & 0 & \frac{2}{\pi} \ln R
\end{array}\right)
$$


and

$$
K\left(z ; i_{B}\right)=I(z)\left(\begin{array}{cc}
-i \tilde{\gamma}^{B} & 0 \\
0 & R^{-1}{ }_{i}{ }^{j}
\end{array}\right)
$$

gives the realization of the transformation, $z \stackrel{i_{B}}{\longrightarrow} z^{\prime}$, given in eq.(3.71)

$$
Z\left(z^{\prime}\right)=G\left(i_{B}\right)^{-1} Z(z) K\left(z ; i_{B}\right)
$$

From eq.(3.24) $L\left(z ; i_{B}\right) \equiv \tilde{\mathrm{x}}_{+}^{-1} \tilde{\gamma}^{B}$ satisfies eq. (4.53).

Eq.(1.81) induces

$$
\bar{Z}\left(z^{\prime}\right)=\bar{K}\left(z ; i_{B}\right) \bar{Z}(z) G\left(i_{B}\right)
$$

where

$$
\bar{K}\left(z ; i_{B}\right)=\left(\begin{array}{cc}
-i \tilde{\gamma}^{B} & 0 \\
0 & R
\end{array}\right) \bar{I}(z)
$$

Note that $G\left(i_{B}\right)^{2}=-1$ and $K\left(z ; i_{B}\right) K\left(z^{\prime} ; i_{B}\right)=-1$.

\subsection{Functions of Three Points}

In a similar fashion to eq. (4.22), a corresponding function $\tilde{F}(\tilde{z})$ for $\tilde{z}^{\tilde{\mathcal{A}}}=\left(y^{A}, \phi^{i}\right) \in \mathbb{R}_{-}^{6 \mid 8 N}$ is given by

$$
\tilde{F}(\tilde{z})=\left(\begin{array}{cc}
-i y_{+} & 2 \phi^{j} \\
2 \bar{\phi}_{i} & \delta_{i}{ }^{j}
\end{array}\right)
$$

With this definition we may write

$$
\overline{\tilde{Z}}\left(\widetilde{z_{31}}\right) \tilde{Z}\left(\widetilde{z_{21}}\right)=\tilde{F}\left(\widetilde{\mathbb{Z}_{1}}\right)=\left(\begin{array}{cc}
-i Y_{1+} & 2 \Phi_{1}^{j} \\
2 \bar{\Phi}_{1 i} & \delta_{i}{ }^{j}
\end{array}\right)
$$

where

$$
\begin{gathered}
\mathrm{Y}_{1+}=\mathcal{X}_{31}^{-1} \mathcal{X}_{32} \mathcal{X}_{12}^{-1} \\
\Phi_{1}^{i}=i\left(\mathcal{X}_{31}^{-1} \theta_{31}^{i}-\mathcal{X}_{21}^{-1} \theta_{21}^{i}\right)
\end{gathered}
$$

with

$$
\bar{\Phi}_{1 i}=\Phi_{1}^{i \dagger} \gamma^{0}=\Phi_{1}^{j} \mathcal{E}_{j i}=i\left(\bar{\theta}_{31 i} \mathcal{X}_{13}^{-1}-\bar{\theta}_{21 i} \mathcal{X}_{12}^{-1}\right)
$$

Using

$$
\mathcal{X}_{12}+\mathcal{X}_{23}=\mathcal{X}_{13}+4 i \theta_{12}^{i} \bar{\theta}_{23 i}
$$

one can show

$$
\begin{gathered}
\mathrm{Y}_{1-}=\mathrm{Y}_{1+}-4 i \Phi_{1}^{i} \bar{\Phi}_{1 i}=-\mathcal{X}_{21}^{-1} \mathcal{X}_{23} \mathcal{X}_{13}^{-1}=-\mathrm{Y}_{1+}^{t} \\
\mathrm{Y}_{1}=\frac{1}{2}\left(\mathrm{Y}_{1+}+\mathrm{Y}_{1-}\right)=-\mathrm{Y}_{1}^{t}=Y_{1}^{A} \gamma_{A}
\end{gathered}
$$


Hence we may define $\widetilde{\mathbb{Z}} \widetilde{\mathcal{A}}^{\tilde{\mathcal{A}}}=\left(Y_{1}^{A}, \Phi_{1}^{i}\right) \in \mathbb{R}_{-}^{6 \mid 8 N}$.

It is evident from eqs. (4.86, 4.89) that under $z_{2} \leftrightarrow z_{3}, \widetilde{\mathbb{Z}_{1}} \rightarrow-\widetilde{\mathbb{Z}_{1}}$.

From eq.(4.78), $\tilde{F}\left(\widetilde{\mathbb{Z}}_{1}\right)$ transforms infinitesimally as

$$
\delta \tilde{F}\left(\widetilde{\mathbb{Z}_{1}}\right)=\left(\begin{array}{cc}
\tilde{\hat{\omega}}\left(z_{1}\right)-\frac{1}{2} \hat{\lambda}\left(z_{1}\right) & 0 \\
0 & \hat{T}\left(z_{1}\right)
\end{array}\right) \tilde{F}\left(\widetilde{\mathbb{Z}_{1}}\right)-\tilde{F}\left(\widetilde{\mathbb{Z}_{1}}\right)\left(\begin{array}{cc}
\hat{\omega}\left(z_{1}\right)+\frac{1}{2} \hat{\lambda}\left(z_{1}\right) & 0 \\
0 & \hat{T}\left(z_{1}\right)
\end{array}\right)
$$

and hence for finite transformations

$$
\tilde{F}\left(\widetilde{\mathbb{Z}}_{1}{ }^{\prime}\right)=\left(\begin{array}{cc}
L\left(z_{1} ; g\right)^{-1} & 0 \\
0 & U\left(z_{1} ; g\right)^{-1}
\end{array}\right) \tilde{F}\left(\widetilde{\mathbb{Z}_{1}}\right)\left(\begin{array}{cc}
L\left(z_{1} ; g\right)^{-1 t} & 0 \\
0 & U\left(z_{1} ; g\right)
\end{array}\right)
$$

Thus $\widetilde{\mathbb{Z}_{1}}$ transforms homogeneously at $z_{1}$. Explicitly we have from eq.(4.91)

$$
\begin{gathered}
\mathrm{Y}_{1+}^{\prime}=L\left(z_{1} ; g\right)^{-1} \mathrm{Y}_{1+} L\left(z_{1} ; g\right)^{-1 t} \\
\Phi_{1}^{i i}=L\left(z_{1} ; g\right)^{-1} \Phi_{1}^{j} U_{j}{ }^{i}\left(z_{1} ; g\right) \quad \bar{\Phi}_{1 i}^{\prime}=U^{-1}{ }_{i}{ }^{j}\left(z_{1} ; g\right) \bar{\Phi}_{1 j} L\left(z_{1} ; g\right)^{-1 t}
\end{gathered}
$$

$\mathrm{Y}_{1-}$ also transforms in the same way as $\mathrm{Y}_{1+}$ in eq.(4.92).

In a similar fashion to eq.(4.24) we get

$$
\operatorname{sdet} \tilde{F}\left(\widetilde{\mathbb{Z}_{1}}\right)=\operatorname{det} Y_{1+}=\operatorname{det} Y_{1-}=\frac{\operatorname{det} \mathcal{X}_{32}}{\operatorname{det} \mathcal{X}_{31} \operatorname{det} \mathcal{X}_{12}}
$$

It is useful to consider $\widetilde{\mathbb{Z}_{1}}=\left(Y_{1}^{A}, \Phi_{1}^{i}\right) \stackrel{i^{-1}}{\longrightarrow} \mathbb{Z}_{1}=\left(X_{1}^{A}, \Theta_{1}^{i}\right)$, where $i^{-1}$ is the inverse of superinversion given in eq. (3.62)

$$
\tilde{\mathrm{X}}_{1 \pm}=-\mathrm{Y}_{1 \pm}^{-1} \quad \Theta_{1}^{i}=-i \mathrm{Y}_{1-}^{-1} \Phi_{1}^{i}
$$

By taking cyclic permutations of $z_{1}, z_{2}, z_{3}$ in eq. $\left(4.86\right.$ ) we may define $\widetilde{\mathbb{Z}_{2}}, \widetilde{\mathbb{Z}_{3}}$ and hence $\mathbb{Z}_{2}, \mathbb{Z}_{3}$. We find $\mathbb{Z}_{2}, \mathbb{Z}_{3}$ are related to $\mathbb{Z}_{1}$ in a simple form

$$
\begin{aligned}
& F\left(\mathbb{Z}_{2}\right)=\left(\begin{array}{cc}
i \mathcal{X}_{21} & 0 \\
0 & V\left(z_{21}\right)
\end{array}\right) \tilde{F}\left(-\widetilde{\mathbb{Z}_{1}}\right)\left(\begin{array}{cc}
i \mathcal{X}_{12} & 0 \\
0 & V\left(z_{21}\right)^{-1}
\end{array}\right) \\
& \tilde{F}\left(\widetilde{\mathbb{Z}_{3}}\right)=\left(\begin{array}{cc}
-i \mathcal{X}_{13}^{-1} & 0 \\
0 & V\left(z_{31}\right)
\end{array}\right) F\left(-\mathbb{Z}_{1}\right)\left(\begin{array}{cc}
-i \mathcal{X}_{31}^{-1} & 0 \\
0 & V\left(z_{13}\right)
\end{array}\right)
\end{aligned}
$$

where $F$ is given in eq.(4.22).

Explicitly we have

$$
\begin{array}{ll}
\tilde{\mathrm{X}}_{2+}=\mathcal{X}_{21} \mathrm{Y}_{1-} \mathcal{X}_{12} & \Theta_{2}^{i}=-i \mathcal{X}_{21} \Phi_{1}^{j} V_{j}{ }^{i}\left(z_{12}\right) \\
\mathrm{Y}_{3+}=\mathcal{X}_{13}^{-1} \tilde{\mathrm{X}}_{1-} \mathcal{X}_{31}^{-1} & \Phi_{3}^{i}=i \mathcal{X}_{13}^{-1} \Theta_{1}^{j} V_{j}{ }^{i}\left(z_{13}\right)
\end{array}
$$


If we define a function $\tilde{V}(\tilde{z}) \in \operatorname{Sp}(N)$ for $\tilde{z} \in \mathbb{R}_{-}^{6 \mid 8 N}$, analogous to $V(z)$ in eq.(4.26), by

$$
\tilde{V}_{i}{ }^{j}(\tilde{z})=\delta_{i}{ }^{j}+4 i \bar{\phi}_{i} Y_{-}^{-1} \phi^{j}
$$

then one can show

$$
\tilde{V}\left(\widetilde{\mathbb{Z}_{1}}\right)=V\left(z_{13}\right) V\left(z_{32}\right) V\left(z_{21}\right)
$$

$\tilde{V}\left(\widetilde{\mathbb{Z}_{1}}\right)$ transforms as

$$
\tilde{V}\left(\widetilde{\mathbb{Z}}_{1}^{\prime}\right)=U^{-1}\left(z_{1} ; g\right) V\left(\widetilde{\mathbb{Z}_{1}}\right) U\left(z_{1} ; g\right)
$$

Note also that with $z \stackrel{i}{\longrightarrow} \tilde{z}$

$$
\tilde{V}(\tilde{z})=V(z)^{-1}
$$

\section{$5 \quad$ Reduction to Four Dimensions}

The reduction of the conformal group to four-dimensions may be defined as the subgroup such that

$$
a^{A}=0 \quad b^{A}=0 \quad \omega^{\mu A}=0 \quad A=4,5
$$

where $\mu=0,1,2,3$.

In the superconformal case, from eq.(3.78), it is necessary therefore to require

$$
\bar{\varepsilon}_{i} \gamma^{A} \varepsilon^{\prime i}=0 \quad \bar{\rho}_{i} \tilde{\gamma}^{A} \rho^{\prime i}=0 \quad \bar{\varepsilon}_{i} \gamma^{[A} \tilde{\gamma}^{\mu]} \rho^{i}=0 \quad A=4,5
$$

To solve this, we first decompose $\varepsilon^{i}=\varepsilon_{+}^{i}+\varepsilon_{-}^{i}$ where

$$
i \tilde{\gamma}_{4} \gamma_{5} \varepsilon_{ \pm}^{i}= \pm \varepsilon_{ \pm}^{i}
$$

Defining $\bar{\varepsilon}_{ \pm i}=\left(\varepsilon_{ \pm}^{i}\right)^{\dagger} \tilde{\gamma}^{0}$ as in eq. (2.21) we have

$$
i \tilde{\gamma}_{4} \gamma_{5} \bar{\varepsilon}_{ \pm i}^{t}=\mp \bar{\varepsilon}_{ \pm i}^{t}
$$

Since $\tilde{\gamma}^{0} \gamma^{1} \tilde{\gamma}^{2} \gamma^{3} \tilde{\gamma}^{4} \gamma^{5}=-1, \varepsilon_{ \pm}^{i}$ are chiral spinors in four-dimensions. We may also decompose $\rho^{i}$ in a similar fashion as $\rho^{i}=\rho_{+}^{i}+\rho_{-}^{i}$ where now $i \gamma_{4} \tilde{\gamma}_{5} \rho_{ \pm}^{i}= \pm \rho_{ \pm}^{i}$.

Since $i \tilde{\gamma}_{4} \gamma_{5}$ satisfies

$$
\gamma^{A}\left(i \tilde{\gamma}_{4} \gamma_{5}\right)= \pm\left(i \tilde{\gamma}_{4} \gamma_{5}\right)^{t} \gamma^{A} \quad\left\{\begin{array}{l}
+: A=4,5 \\
-: A=\mu=0,1,2,3
\end{array}\right.
$$

eq.(5.2) becomes

$$
\begin{aligned}
& \bar{\varepsilon}_{+i} \gamma^{A} \varepsilon_{-}^{i i}+\bar{\varepsilon}_{-i} \gamma^{A} \varepsilon_{+}^{i i}=\bar{\rho}_{+i} \tilde{\gamma}^{A} \rho_{-}^{i i}+\bar{\rho}_{-i} \tilde{\gamma}^{A} \rho_{+}^{\prime i}=0 \\
& \bar{\varepsilon}_{+i} \gamma^{[A} \tilde{\gamma}^{\mu]} \rho_{-}^{i}+\bar{\varepsilon}_{-i} \gamma^{[A} \tilde{\gamma}^{\mu]} \rho_{+}^{i}=0 \quad A=4,5
\end{aligned}
$$


To solve the conditions (5.6) we require $\bar{\varepsilon}_{+i}, \bar{\rho}_{+i}$ to be orthogonal to $\varepsilon_{-}^{i}, \rho_{-}^{i}$ when contracting the indices $i$. The solutions for $\varepsilon_{ \pm}^{i}$ define spaces $V_{ \pm}$where $\varepsilon_{ \pm}^{i} \in V_{ \pm}$and since, by virtue of eq.(5.4), complex conjugation interchanges $V_{+}$and $V_{-}$, we must have $\operatorname{dim} V_{+}=\operatorname{dim} V_{-}$. The maximal solution of eq. (5.6) occurs when we reduce half of the degrees of freedom for the spinors so that $\operatorname{dim} V_{+}=\operatorname{dim} V_{-}=N$. Using a basis where

$$
i \tilde{\gamma}_{4} \gamma_{5},-i \gamma_{4} \tilde{\gamma}_{5}=\left(\begin{array}{cc}
1 & 0 \\
0 & -1
\end{array}\right)
$$

the solution can be written, using the freedom of $\operatorname{Sp}(N)$ transformations, in the form

$$
\varepsilon^{i}=\left(\begin{array}{cc}
\bar{\varepsilon}_{a} & 0 \\
0 & \varepsilon^{a t}
\end{array}\right) \quad \rho^{i}=\left(\begin{array}{cc}
0 & \bar{\rho}^{a t} \\
\rho_{a} & 0
\end{array}\right) \quad a=1,2, \cdots, N
$$

and so following eqs. 3.34, 3.39)

$$
\bar{\varepsilon}_{i}=\left(\begin{array}{cc}
0 & \varepsilon^{a} \\
-\bar{\varepsilon}_{a}^{t} & 0
\end{array}\right) \quad \bar{\rho}_{i}=\left(\begin{array}{cc}
\bar{\rho}^{a} & 0 \\
0 & -\rho_{a}^{t}
\end{array}\right)
$$

The representation】 of $\gamma^{\mu}, \tilde{\gamma}^{\mu}$ may be chosen such that $\bar{\varepsilon}_{a}^{\dot{\alpha}}=\varepsilon^{a \alpha \dagger}, \bar{\rho}_{\dot{\alpha}}^{a}=\rho_{a \alpha}^{\dagger} \alpha, \dot{\alpha}=1,2$. Since, from eq.(3.78), $\varepsilon^{j} T_{j}{ }^{i}, \rho^{j} T_{j}{ }^{i}$ should remain of the form (5.8), it is necessary to restrict the $\operatorname{Sp}(N)$ generators, $T_{i}^{j}$ to the form

$$
T_{j}^{i}=\left(\begin{array}{cc}
t^{a}{ }_{b}+i \phi \delta^{a}{ }_{b} & 0 \\
0 & -\left(t^{t}\right)_{a}^{b}-i \phi \delta_{a}^{b}
\end{array}\right)
$$

where $t \in \mathrm{su}(N), \phi \in S^{1}$. With this form $\mathrm{Sp}(N) \rightarrow \mathrm{SU}(N) \times \mathrm{U}(1)$

After reduction to four-dimensions, the matrix representation of superconformal algebra, $M$, given in eq. (3.79) decomposes as

$$
M=M_{+}+M_{-} \quad M_{-}=-C^{-1} M_{+}^{t} C
$$

where, with $\omega_{45}=-\psi, w=\frac{1}{4} \omega_{\mu \nu} \tilde{\sigma}^{\mu} \sigma^{\nu}, \tilde{w}=\frac{1}{4} \omega_{\mu \nu} \sigma^{\mu} \tilde{\sigma}^{\nu}$,

$$
M_{+}=\left(\begin{array}{ccc}
\left(w+\frac{1}{2} \lambda+i \frac{1}{2} \psi\right) p_{+} & -i a \cdot \tilde{\sigma} \tau_{+} & 2 \bar{\varepsilon}_{b} p_{+} \\
-i b \cdot \sigma \tau_{-} & \left(\tilde{w}-\frac{1}{2} \lambda+i \frac{1}{2} \psi\right) p_{-} & 2 \rho_{b} \tau_{-} \\
2 \bar{\rho}^{a} p_{+} & 2 \varepsilon^{a} \tau_{+} & \left(t^{a}{ }_{b}+i \phi \delta_{b}^{a}{ }_{b}\right) p_{+}
\end{array}\right)
$$

\footnotetext{
${ }^{7}$ See Appendix A for a particular choice of representation for $\gamma^{\mu}, \tilde{\gamma}^{\mu}$ compatible with eq.(5.9).
} 
and we define

$$
p_{+}=\left(\begin{array}{cc}
1 & 0 \\
0 & 0
\end{array}\right) \quad p_{-}=\left(\begin{array}{cc}
0 & 0 \\
0 & 1
\end{array}\right) \quad \tau_{+}=\left(\begin{array}{cc}
0 & 1 \\
0 & 0
\end{array}\right) \quad \tau_{-}=\left(\begin{array}{cc}
0 & 0 \\
1 & 0
\end{array}\right)
$$

Since $p_{+}^{2}=\tau_{+} \tau_{-}=p_{+}, p_{-}^{2}=\tau_{-} \tau_{+}=p_{-}, p_{+} \tau_{+}=\tau_{+} p_{-}=\tau_{+}, p_{-} \tau_{-}=\tau_{-} p_{+}=\tau_{-}$, the form of $M_{+}$is closed under multiplication and also $\left[M_{+}, M_{-}^{\prime}\right]=0$. Hence $M_{+}$alone defines the four-dimensional superconformal algebra, and it can be naturally reduced to the $(4+N) \times(4+N)$ matrix, $\mathcal{M}$

$$
\mathcal{M}=\left(\begin{array}{ccc}
w+\frac{1}{2} \lambda+i \frac{1}{2} \psi & -i a \cdot \tilde{\sigma} & 2 \bar{\varepsilon}_{b} \\
-i b \cdot \sigma & \tilde{w}-\frac{1}{2} \lambda+i \frac{1}{2} \psi & 2 \rho_{b} \\
2 \bar{\rho}^{a} & 2 \varepsilon^{a} & t^{a}{ }_{b}+i \frac{2}{N} \psi \delta^{a}{ }_{b}
\end{array}\right)
$$

where we impose $\operatorname{str} \mathcal{M}=\operatorname{str} M_{+}=i(2 \psi-N \phi)=0$, removing a $\mathrm{U}(1)$ factor, which is consistent with $\operatorname{str}\left[\mathcal{M}, \mathcal{M}^{\prime}\right]=0$. For general $N$ the $R$-symmetry group is $\mathrm{SU}(N) \times \mathrm{U}(1)$. When $N=4$, however, it is evident from eq.(5.14) that $\mathcal{M}=\mathcal{M}_{0}+i \frac{1}{2} \psi 1$ where $\mathcal{M}_{0}$ is of the form (5.14) with $\psi=0$, so that $\psi$ parameterizes a $\mathrm{U}(1)$ invariant subalgebra. Hence in this case we may set $\psi=0$ and reduce $\mathcal{M}$ to $\mathcal{M}_{0}$. The associated $\mathrm{U}(1)$ symmetry is an ideal of four-dimensional $N=4$ superconformal symmetry, so that the $R$-symmetry group is just $\mathrm{SU}(4)$.

From eq.(3.82) the hermiticity condition becomes

$$
\mathcal{B M B}^{-1}=-\mathcal{M}^{\dagger} \quad \mathcal{B}=\left(\begin{array}{ccc}
0 & 1 & 0 \\
1 & 0 & 0 \\
0 & 0 & -1
\end{array}\right)
$$

\subsection{Reduction of Superspace}

The reduction of $\mathbb{R}_{+}^{6 \mid 8 N}$ superspace to four-dimensions is defined for $z^{\mathcal{A}}=\left(x^{A}, \theta^{i}\right)$ by setting $x^{4}=x^{5}=0$ and, as in eqs. $(5.8,5.9)$, restricting $\theta^{i}$ to the form

$$
\theta^{i}=\left(\begin{array}{cc}
\bar{\theta}_{a} & 0 \\
0 & \theta^{a t}
\end{array}\right) \quad \Longrightarrow \quad \bar{\theta}_{i}=\left(\begin{array}{cc}
0 & \theta^{a} \\
-\bar{\theta}_{a}^{t} & 0
\end{array}\right)
$$

Hence $z^{\mathcal{A}} \rightarrow z^{\mathcal{N}}=\left(x^{\mu}, \theta^{a \alpha}, \bar{\theta}_{a}^{\dot{\alpha}}\right) \in \mathbb{R}^{4 \mid 4 N}$ and the six-dimensional infinitesimal supersymmetric interval is furthermore projected into four-dimensions, $e^{\mathcal{A}}(z) \rightarrow e^{\mathcal{N}}(z)$, where

$$
e^{\mathcal{N}}(z)=\left(e^{\mu}(z), \mathrm{d} \theta^{a \alpha}, \mathrm{d} \bar{\theta}_{a}^{\dot{\alpha}}\right) \quad e^{\mu}(z)=\mathrm{d} x^{\mu}+i \mathrm{~d} \theta^{a} \sigma^{\mu} \bar{\theta}_{a}-i \theta^{a} \sigma^{\mu} \mathrm{d} \bar{\theta}_{a}
$$


$\tilde{\mathrm{x}}_{ \pm}$defined in eq.(3.23) is of the form

$$
\tilde{\mathrm{x}}_{ \pm}=\left(\begin{array}{cc}
0 & x_{ \pm} \cdot \tilde{\sigma} \\
-\left(x_{\mp} \cdot \tilde{\sigma}\right)^{t} & 0
\end{array}\right) \quad x_{ \pm}^{\mu}=x^{\mu} \mp i \theta^{a} \sigma^{\mu} \bar{\theta}_{a}
$$

Note that

$$
x_{ \pm} \cdot \tilde{\sigma}=x \cdot \tilde{\sigma} \pm 2 i \bar{\theta}_{a} \theta^{a}
$$

The four-dimensional superconformal transformations are now realized by restricting the six-dimensional $(N, 0)$ superconformal transformations as in $M \rightarrow \mathcal{M}$ with the group of dimensions $\left(15+N^{2} \mid 8 N\right)$ if $N \neq 4$. We then get $N$-extended superconformal transformations in four-dimensions from eqs.(3.41, 3.42a)

$$
\begin{aligned}
& \delta \theta^{a}=\varepsilon^{a}+\frac{1}{2}(\lambda+i \Omega) \theta^{a}-\theta^{a} \tilde{w}+t_{b}^{a} \theta^{b}+\theta^{a} b \cdot \sigma x_{+} \cdot \tilde{\sigma}-i \bar{\rho}^{a} x_{+} \cdot \tilde{\sigma}-4\left(\theta^{a} \rho_{b}\right) \theta^{b} \\
& \delta x_{+} \cdot \tilde{\sigma}=x_{+} \cdot \tilde{\sigma} b \cdot \sigma x_{+} \cdot \tilde{\sigma}-4 x_{+} \cdot \tilde{\sigma} \rho_{a} \theta^{a}+\lambda x_{+} \cdot \tilde{\sigma}+w x_{+} \cdot \tilde{\sigma}-x_{+} \cdot \tilde{\sigma} \tilde{w}+4 i \bar{\varepsilon}_{a} \theta^{a}+a \cdot \tilde{\sigma}
\end{aligned}
$$

where $\Omega=\left(\frac{4}{N}-1\right) \psi$. When $N=4$, we get $\Omega=0$ and the transformations are independent of $\psi$ which is consistent with the reduction of the superconformal algebra in this case.

$Z(z)$ and $\bar{Z}(z)$ defined by eqs. (4.14,4.18) can also be decomposed in a similar fashion to eqs.(5.11, 5.12) by writing

$$
Z(z)=Z_{+}(z)+Z_{-}(z) \quad \bar{Z}(z)=\bar{Z}_{+}(z)+\bar{Z}_{-}(z)
$$

where

$$
Z_{+}(z)=\left(\begin{array}{cc}
-i x_{+} \cdot \tilde{\sigma} \tau_{+} & 2 \bar{\theta}_{b} p_{+} \\
p_{-} & 0 \\
2 \theta^{a} \tau_{+} & \delta^{a}{ }_{b} p_{+}
\end{array}\right) \quad \bar{Z}_{+}(z)=\left(\begin{array}{ccc}
p_{+} & i x_{-} \cdot \tilde{\sigma} \tau_{+} & -2 \bar{\theta}_{b} p_{+} \\
0 & -2 \theta^{a} \tau_{+} & \delta_{b}^{a} p_{+}
\end{array}\right)
$$

and $Z_{-}(z), \bar{Z}_{-}(z)$ are given by

$$
Z_{-}(z)=C^{-1} \bar{Z}_{+}^{t}(z)\left(\begin{array}{cc}
1 & 0 \\
0 & \overline{\mathcal{E}}
\end{array}\right) \quad \bar{Z}_{-}(z)=\left(\begin{array}{cc}
1 & 0 \\
0 & -\overline{\mathcal{E}}
\end{array}\right) Z_{+}(z)^{t} C
$$

Just as $M_{+} \rightarrow \mathcal{M}$, we can similarly have

$$
\begin{aligned}
& Z_{+}(z) \rightarrow \mathcal{Z}(z)=\left(\begin{array}{cc}
-i x_{+} \cdot \tilde{\sigma} & 2 \bar{\theta}_{b} \\
1 & 0 \\
2 \theta^{a} & \delta^{a}{ }_{b}
\end{array}\right) \\
& \bar{Z}_{+}(z) \rightarrow \overline{\mathcal{Z}}(z)=\left(\begin{array}{ccc}
1 & i x_{-} \cdot \tilde{\sigma} & -2 \bar{\theta}_{b} \\
0 & -2 \theta^{a} & \delta^{a}{ }_{b}
\end{array}\right)=\left(\begin{array}{cc}
1 & 0 \\
0 & -1
\end{array}\right) \mathcal{Z}(z)^{\dagger} \mathcal{B}
\end{aligned}
$$


Infinitesimally $\mathcal{Z}(z), \overline{\mathcal{Z}}(z)$ transform, from eqs.(4.48, 4.55), as

$$
\begin{aligned}
& \delta \mathcal{Z}(z)=\mathcal{M} \mathcal{Z}(z)-\mathcal{Z}(z) \mathcal{H}(z) \\
& \delta \overline{\mathcal{Z}}(z)=\overline{\mathcal{H}}(z) \overline{\mathcal{Z}}(z)-\overline{\mathcal{Z}}(z) \mathcal{M}
\end{aligned}
$$

where

$$
\begin{aligned}
& \mathcal{H}(z)=\left(\begin{array}{cc}
\tilde{\hat{w}}(z)-\frac{1}{2} \hat{\lambda}(z)+i \frac{1}{2} \hat{\psi}(z) & 2 \hat{\rho}_{b}(z) \\
0 & \hat{t}^{a}{ }_{b}(z)+i \frac{2}{N} \hat{\psi}(z) \delta^{a}{ }_{b}
\end{array}\right) \\
& \overline{\mathcal{H}}(z)=\left(\begin{array}{cc}
\hat{w}(z)+\frac{1}{2} \hat{\lambda}(z)+i \frac{1}{2} \hat{\psi}(z) & 0 \\
22 \hat{\hat{\rho}}^{a}(z) & \hat{t}^{a}{ }_{b}(z)+i \frac{2}{N} \hat{\psi}(z) \delta_{b}^{a}
\end{array}\right)
\end{aligned}
$$

with

$$
\begin{aligned}
& \tilde{\hat{w}}(z)-\frac{1}{2} \hat{\lambda}(z)+i \frac{1}{2} \hat{\psi}(z)=\tilde{w}+4 \rho_{a} \theta^{a}-b \cdot \sigma x_{+} \cdot \tilde{\sigma}-\frac{1}{2} \lambda+i \frac{1}{2} \psi \\
& \hat{w}(z)+\frac{1}{2} \hat{\lambda}(z)+i \frac{1}{2} \hat{\psi}(z)=w-4 \bar{\theta}_{a} \bar{\rho}^{a}+x_{-} \cdot \tilde{\sigma} b \cdot \sigma+\frac{1}{2} \lambda+i \frac{1}{2} \psi=-\left(\tilde{\hat{w}}(z)-\frac{1}{2} \hat{\lambda}(z)+i \frac{1}{2} \hat{\psi}(z)\right)^{\dagger} \\
& \hat{t}^{a}{ }_{b}(z)+i \frac{2}{N} \hat{\psi}(z) \delta_{b}^{a}=t_{b}^{a}+4 i \theta^{a} b \cdot \sigma \bar{\theta}_{b}+4\left(\bar{\rho}^{a} \bar{\theta}_{b}-\theta^{a} \rho_{b}\right)+i \frac{2}{N} \psi \delta_{b}^{a} \\
& \hat{\rho}_{a}(z)=\rho_{a}-i b \cdot \sigma \bar{\theta}_{a} \quad \overline{\hat{\rho}}^{a}(z)=\bar{\rho}^{a}+i \theta^{a} b \cdot \sigma=\hat{\rho}_{a}(z)^{\dagger}
\end{aligned}
$$

In general, with $z^{\mathcal{A}} \rightarrow z^{\mathcal{N}} \in \mathbb{R}^{4 \mid 4 N}, F(z)$ defined in eq. (4.22) can also be decomposed as

$$
F(z)=F_{+}(z)+F_{-}(z)
$$

where

$$
\begin{aligned}
& F_{+}(z)=\left(\begin{array}{cc}
-i x_{+} \cdot \tilde{\sigma} \tau_{+} & 2 \bar{\theta}_{b} p_{+} \\
2 \theta^{a} \tau_{+} & \delta^{a}{ }_{b} p_{+}
\end{array}\right) \\
& F_{-}(z)=\left(\begin{array}{cc}
1 & 0 \\
0 & -\overline{\mathcal{E}}
\end{array}\right) F_{+}(-z)^{t}\left(\begin{array}{cc}
1 & 0 \\
0 & -\mathcal{E}
\end{array}\right)
\end{aligned}
$$

Analogous to eqs. $4.22,4.25,4.26)$ we define for any $z^{\mathcal{N}}=\left(x^{\mu}, \theta^{a \alpha}, \bar{\theta}_{a}^{\dot{\alpha}}\right) \in \mathbb{R}^{4 \mid 4 N}$

$$
\mathcal{F}(z)=\left(\begin{array}{cc}
-i x_{+} \cdot \tilde{\sigma} & 2 \bar{\theta}_{b} \\
2 \theta^{a} & \delta_{b}^{a}
\end{array}\right)
$$

so that $F_{+}(z) \rightarrow \mathcal{F}(z)$. Furthermore

$$
\left(\begin{array}{cc}
1 & 0 \\
-2 i \theta^{a}\left(x_{+} \cdot \tilde{\sigma}\right)^{-1} & 1
\end{array}\right) \mathcal{F}(z)\left(\begin{array}{cc}
1 & -2 i\left(x_{+} \cdot \tilde{\sigma}\right)^{-1} \bar{\theta}_{b} \\
0 & 1
\end{array}\right)=\left(\begin{array}{cc}
-i x_{+} \cdot \tilde{\sigma} & 0 \\
0 & v_{b}^{a}(-z)
\end{array}\right)
$$


defines

$$
v_{b}^{a}(z)=\delta_{b}^{a}+4 i \frac{1}{x_{-}^{2}} \theta^{a} x_{-} \cdot \sigma \bar{\theta}_{b}
$$

which satisfies

$$
v_{b}^{a}(-z)=v_{b}^{-1 a}(z)=v_{b}^{\dagger a}(z)
$$

The superdeterminant of $\mathcal{F}(z)$ is given by

$$
\operatorname{sdet} \mathcal{F}(z)=-x_{-}^{2}
$$

and also from eq.(5.31)

$$
\operatorname{det} v(z)=\frac{x_{-}^{2}}{x_{+}^{2}}
$$

In the above, we considered the reduction $\mathbb{R}_{+}^{6 \mid 8 N} \rightarrow \mathbb{R}^{4 \mid 4 N}$. Alternatively we may consider the reduction of $\mathbb{R}_{-}^{6 \mid 8 N}$ superspace. For $\tilde{z}^{\tilde{\mathcal{A}}}=\left(y^{A}, \phi^{i}\right) \in \mathbb{R}_{-}^{6 \mid 8 N}$ we set $y^{4}=y^{5}=0$ and, analogous to eq. (5.16),

$$
\phi^{i}=\left(\begin{array}{cc}
0 & \bar{\phi}^{a t} \\
\phi_{a} & 0
\end{array}\right) \quad \Longrightarrow \quad \bar{\phi}_{i}=\left(\begin{array}{cc}
\bar{\phi}^{a} & 0 \\
0 & -\phi_{a}^{t}
\end{array}\right)
$$

Hence $\tilde{z}^{\tilde{\mathcal{A}}} \rightarrow \tilde{z}^{\tilde{\mathcal{N}}}=\left(y^{\mu}, \phi_{a}, \bar{\phi}^{a}\right)$.

After reducing to four-dimensions, $\mathbb{R}_{ \pm}^{6 \mid 8 N} \rightarrow \mathbb{R}^{4 \mid 4 N}$, the superspaces are no longer inequivalent. This is apparent by using the reflection transformation $r_{5}$ defined by eqs.(3.68, 3.69) with the $\operatorname{Sp}(N)$ transformation $R$ given by

$$
R_{i}{ }^{j}=\left(\begin{array}{cc}
0 & -\zeta^{a b} \\
\bar{\zeta}_{a b} & 0
\end{array}\right) \quad \zeta^{a b} \bar{\zeta}_{b c}=\delta_{c}^{a} \quad \zeta^{a b}=\zeta^{b a} \quad \bar{\zeta}_{a b}=\left(\zeta^{a b}\right)^{*}
$$

which satisfies eq.(3.73). Letting now $\mathbb{R}_{-}^{6 \mid 8 N} \stackrel{r_{5}^{-1}}{\longrightarrow} \mathbb{R}_{+}^{6 \mid 8 N} \longrightarrow \mathbb{R}^{4 \mid 4 N}$, we get $\tilde{z}^{\tilde{\mathcal{A}}} \rightarrow z^{\mathcal{N}}=$ $\left(x^{\mu}, \theta^{a}, \bar{\theta}_{a}\right)$ where $x^{\mu}=y^{\mu}$ and

$$
\theta^{a}=-\zeta^{a b} \phi_{b}^{t} \epsilon^{-1} \quad \bar{\theta}_{a}=\bar{\epsilon}^{-1} \bar{\phi}^{b t} \bar{\zeta}_{b a}
$$

In a similar fashion to eq.(5.18), $\mathrm{y}_{ \pm}=\mathrm{y} \pm 2 i \phi^{i} \bar{\phi}_{i}$ reduces to

$$
\mathrm{y}_{ \pm}=\left(\begin{array}{cc}
0 & -\left(y_{ \pm} \cdot \sigma\right)^{t} \\
y_{\mp} \cdot \sigma & 0
\end{array}\right) \quad y_{ \pm}^{\mu}=y^{\mu} \pm i \bar{\phi}^{a} \tilde{\sigma}^{\mu} \phi_{a}=x_{ \pm}^{\mu}
$$

where we may also write

$$
y_{ \pm} \cdot \sigma=y \cdot \sigma \mp 2 i \phi_{a} \bar{\phi}^{a}
$$


In a similar fashion to eqs.(5.28, 5.29), $\tilde{F}(\tilde{z})$ defined in eq.(4.84) can be decomposed as

$$
\tilde{F}(\tilde{z})=\tilde{F}_{+}(\tilde{z})+\tilde{F}_{-}(\tilde{z})
$$

where

$$
\begin{aligned}
& \tilde{F}_{+}(\tilde{z})=\left(\begin{array}{cc}
-i y_{-} \cdot \sigma \tau_{-} & 2 \phi_{b} \tau_{-} \\
2 \bar{\phi}^{a} p_{+} & \delta^{a}{ }_{b} p_{+}
\end{array}\right) \\
& \tilde{F}_{-}(\tilde{z})=\left(\begin{array}{cc}
1 & 0 \\
0 & -\overline{\mathcal{E}}
\end{array}\right) \tilde{F}_{+}(-\tilde{z})^{t}\left(\begin{array}{cc}
1 & 0 \\
0 & -\mathcal{E}
\end{array}\right)
\end{aligned}
$$

Hence we reduce $\tilde{F}_{+}(\tilde{z})$ to $\tilde{\mathcal{F}}(\tilde{z})$

$$
\tilde{\mathcal{F}}(\tilde{z})=\left(\begin{array}{cc}
-i y_{-} \cdot \sigma & 2 \phi_{b} \\
2 \bar{\phi}^{a} & \delta^{a}{ }_{b}
\end{array}\right)
$$

With eq. 5.38$) \tilde{\mathcal{F}}(\tilde{z})$ is related to $\mathcal{F}(z)$ as

$$
\mathcal{F}(z)=\left(\begin{array}{cc}
\bar{\epsilon}^{-1} & 0 \\
0 & -\zeta
\end{array}\right) \tilde{\mathcal{F}}(-\tilde{z})^{t}\left(\begin{array}{cc}
\epsilon^{-1} & 0 \\
0 & -\bar{\zeta}
\end{array}\right)
$$

\subsection{Superinversion in $\mathbb{R}^{4 \mid 4 N}$}

In the four-dimensional superspace, $\mathbb{R}^{4 \mid 4 N}$, superinversion may be defined by the reduction of $i_{5}$, given by eq.(3.71) with $R$ as in eq.(5.37). This definition gives $z^{\mathcal{N}} \rightarrow z^{\prime \mathcal{N}}=\left(x^{\prime \mu}, \theta^{\prime a \alpha}, \bar{\theta}_{a}^{\prime \dot{\alpha}}\right)$ where

$$
x_{ \pm}^{\prime \mu}=-\frac{x_{\mp}^{\mu}}{x_{\mp}^{2}} \quad \theta^{\prime a t}=-i \frac{1}{x_{-}^{2}} \epsilon^{-1} x_{-} \cdot \sigma \bar{\theta}_{b} \zeta^{b a} \quad \bar{\theta}_{a}^{\prime}=i \frac{1}{x_{+}^{2}} \bar{\epsilon}^{-1} x_{+} \cdot \sigma^{t} \theta^{b t} \bar{\zeta}_{b a}
$$

This may be rewritten as

$$
\begin{aligned}
\theta^{a} & =i \frac{1}{x_{-}^{2}} \tilde{\bar{\theta}}^{a} x_{-} \cdot \tilde{\sigma} & \tilde{\bar{\theta}}^{a} & =-\zeta^{b a} \bar{\theta}_{b}^{t} \bar{\epsilon} \\
\bar{\theta}_{a}^{\prime} & =-i \frac{1}{x_{+}^{2}} x_{+} \cdot \tilde{\sigma} \tilde{\theta}_{a} & \tilde{\theta}_{a} & =\epsilon \theta^{b t} \bar{\zeta}_{b a}
\end{aligned}
$$

which is just the conventional definition of superinversion in four-dimensions.

In six-dimensional superspace the transformation $i_{5}$ belongs to the connected superconformal group and restricting to four-dimensions we may write from eqs.(4.79, 4.80)

$$
G\left(i_{5}\right)^{-1}=G_{+}\left(i_{5}\right)^{-1}+G_{-}\left(i_{5}\right)^{-1} \quad K\left(z ; i_{5}\right)=K_{+}\left(z ; i_{5}\right)+K_{-}\left(z ; i_{5}\right)
$$


where

$$
\begin{gathered}
G_{+}\left(i_{5}\right)^{-1}=\left(\begin{array}{ccc}
0 & \epsilon^{-1} p_{-} & 0 \\
\bar{\epsilon} p_{+} & 0 & 0 \\
0 & 0 & \bar{\zeta}_{a b} \tau_{-}
\end{array}\right) \\
K_{+}\left(z ; i_{5}\right)=\left(\begin{array}{cc}
i\left(\bar{\epsilon} x_{+} \cdot \tilde{\sigma}\right)^{-1} \tau_{-} & -2 i\left(x_{-} \cdot \tilde{\sigma}\right)^{-1} \bar{\theta}_{c} \zeta^{c b} p_{-} \\
0 & v^{a}(z) \zeta^{c b} \tau_{+}
\end{array}\right) \\
v^{a}{ }_{b}(z)=\delta^{a}{ }_{b}+4 i \frac{1}{x_{-}^{2}} \theta^{a} x_{-} \cdot \sigma \bar{\theta}_{b}
\end{gathered}
$$

and $G_{-}\left(i_{5}\right)^{-1}, K_{-}\left(z ; i_{5}\right)$ are given by

$$
G_{-}\left(i_{5}\right)^{-1}=C^{-1} G_{+}\left(i_{5}\right)^{t} C \quad K_{-}\left(z ; i_{5}\right)^{t}=\left(\begin{array}{cc}
\tilde{\gamma}^{0} & 0 \\
0 & \mathcal{E}
\end{array}\right) K_{+}\left(z ; i_{5}\right)^{\dagger}\left(\begin{array}{cc}
\gamma^{0} & 0 \\
0 & \overline{\mathcal{E}}
\end{array}\right)
$$

Then $z \stackrel{i_{5}}{\longrightarrow} z^{\prime}$ is given by

$$
G_{+}\left(i_{5}\right)^{-1} Z_{+}(z) K_{+}\left(z ; i_{5}\right)=Z_{-}\left(z^{\prime}\right)
$$

which can be reduced to

$$
\mathcal{G}(i)^{-1} \mathcal{Z}(z) \mathcal{K}(z ; i)=\left(\begin{array}{cc}
1 & 0 \\
i x_{-}^{\prime} \cdot \tilde{\sigma}^{t} & -2 \theta^{\prime a t} \\
2 \bar{\theta}_{b}^{\prime t} & \delta_{b}{ }^{a}
\end{array}\right)=\overline{\mathcal{Z}}\left(z^{\prime}\right)^{t}
$$

where

$$
\mathcal{G}(i)^{-1}=\left(\begin{array}{ccc}
\bar{\epsilon} & 0 & 0 \\
0 & \epsilon^{-1} & 0 \\
0 & 0 & -\bar{\zeta}_{a b}
\end{array}\right) \quad \mathcal{K}(z ; i)=\left(\begin{array}{cc}
i\left(\bar{\epsilon} x_{+} \cdot \tilde{\sigma}\right)^{-1} & 2 i\left(x_{-} \cdot \tilde{\sigma}\right)^{-1} \bar{\theta}_{c} \zeta^{c b} \\
0 & -v_{c}^{a}(z) \zeta^{c b}
\end{array}\right)
$$

Similarly we have

$$
\overline{\mathcal{K}}(z ; i) \overline{\mathcal{Z}}(z) \mathcal{G}(i)=\mathcal{Z}\left(z^{\prime}\right)^{t}
$$

where

$$
\begin{gathered}
\overline{\mathcal{K}}(z ; i)=\left(\begin{array}{cc}
-i\left(x_{-} \cdot \tilde{\sigma} \epsilon\right)^{-1} & 0 \\
2 i \bar{\zeta}_{a c} \theta^{c}\left(x_{+} \cdot \tilde{\sigma}\right)^{-1} & -\bar{\zeta}_{a c} v^{-1 c}{ }_{b}(z)
\end{array}\right) \\
v^{-1 a}{ }_{b}(z)=v_{b}^{\dagger a}(z)=v_{b}^{a}(-z)=\delta_{b}^{a}-4 i \frac{1}{x_{+}^{2}} \theta^{a} x_{+} \cdot \sigma \bar{\theta}_{b}
\end{gathered}
$$




\subsection{Functions of Two-points in $\mathbb{R}^{4 \mid 4 N}$}

For two points, the supersymmetric interval $z_{12}^{\mathcal{A}}$ defined in eq.(4.3) can be reduced $z_{12}^{\mathcal{A}} \rightarrow$ $z_{12}^{\mathcal{N}}=\left(x_{12}^{\mu}, \theta_{12}^{a \alpha}, \bar{\theta}_{12 a}^{\dot{\alpha}}\right)=-z_{21}^{\mathcal{N}}$

$$
x_{12}^{\mu}=x_{1}^{\mu}-x_{2}^{\mu}+i \theta_{1}^{a} \sigma^{\mu} \bar{\theta}_{2 a}-i \theta_{2}^{a} \sigma^{\mu} \bar{\theta}_{1 a}
$$

Now we have from eqs. $5.22,5.29$

$$
\bar{Z}_{+}\left(z_{2}\right) Z_{+}\left(z_{1}\right)=F_{+}\left(z_{12}\right)=\left(\begin{array}{cc}
i x_{\overline{2} 1} \cdot \tilde{\sigma} \tau_{+} & -2 \bar{\theta}_{21 b} p_{+} \\
-2 \theta_{21}^{a} \tau_{+} & \delta^{a}{ }_{b} p_{+}
\end{array}\right)
$$

where

$$
x_{\overline{2} 1}^{\mu}=x_{2-}^{\mu}-x_{1+}^{\mu}-2 i \theta_{1}^{a} \sigma^{\mu} \bar{\theta}_{2 a}=x_{21}^{\mu}+i \theta_{21}^{a} \sigma^{\mu} \bar{\theta}_{21 a}=\left(x_{21}^{\mu}\right)_{-}=-\left(x_{12}^{\mu}\right)_{+}
$$

Similarly we write

$$
x_{\overline{1} 2}^{\mu}=x_{1-}^{\mu}-x_{2+}^{\mu}-2 i \theta_{2}^{a} \sigma^{\mu} \bar{\theta}_{1 a}=x_{12}^{\mu}+i \theta_{12}^{a} \sigma^{\mu} \bar{\theta}_{12 a}=\left(x_{12}^{\mu}\right)_{-}=-\left(x_{21}^{\mu}\right)_{+}
$$

In terms of eqs. (5.24, 5.30) (5.56) is reduced to

$$
\overline{\mathcal{Z}}\left(z_{2}\right) \mathcal{Z}\left(z_{1}\right)=\mathcal{F}\left(z_{12}\right)=\left(\begin{array}{cc}
i x_{\overline{2} 1} \cdot \tilde{\sigma} & -2 \bar{\theta}_{21 b} \\
-2 \theta_{21}^{a} & \delta^{a}{ }_{b}
\end{array}\right)
$$

From eq. (5.34)

$$
\text { sdet } \mathcal{F}\left(z_{12}\right)=-x_{12}^{2}
$$

Infinitesimally $\mathcal{F}\left(z_{12}\right)$ transforms, from eq. (5.25), as

$$
\delta \mathcal{F}\left(z_{12}\right)=\overline{\mathcal{H}}\left(z_{2}\right) \mathcal{F}\left(z_{12}\right)-\mathcal{F}\left(z_{12}\right) \mathcal{H}\left(z_{1}\right)
$$

From eqs. (5.51, 5.53) under superinversion

$$
\overline{\mathcal{K}}\left(z_{2} ; i\right) \mathcal{F}\left(z_{12}\right) \mathcal{K}\left(z_{1} ; i\right)=\mathcal{F}\left(z_{12}^{\prime}\right)^{t}
$$

which gives using eq. (5.59)

$$
\left(x_{2-} \cdot \tilde{\sigma}\right)^{-1} x_{\overline{2} 1} \cdot \tilde{\sigma}\left(x_{1+} \cdot \tilde{\sigma}\right)^{-1}=-x_{12}^{\prime} \cdot \sigma \quad x_{12}^{\prime 2}=\frac{x_{\overline{2} 1}^{2}}{x_{2-}^{2} x_{1+}^{2}}
$$

By considering $\mathcal{F}\left(z_{12}\right)^{-1}$ we may also show

$$
\bar{\zeta} v^{-1}\left(z_{2}\right) v\left(z_{21}\right) v\left(z_{1}\right) \zeta=v\left(z_{21}^{\prime}\right)^{t}
$$




\subsection{Functions of Three-points in $\mathbb{R}^{4 \mid 4 N}$}

For three points, just as in the six-dimensional case, we may define $\widetilde{\mathbb{Z}}_{1}^{\mathcal{N}} \in \mathbb{R}^{4 \mid 4 N}$ which transforms homogeneously at $z_{1} \in \mathbb{R}^{4 \mid 4 N}$. With eq. (4.85)

$$
\tilde{\mathcal{F}}\left(\widetilde{\mathbb{Z}}_{1}\right)=\left(\begin{array}{cc}
-i Y_{1-} \cdot \sigma & 2 \Phi_{1 b} \\
2 \bar{\Phi}_{1}^{a} & \delta_{b}^{a}
\end{array}\right)
$$

where

$$
\begin{aligned}
& Y_{1-} \cdot \sigma=\left(x_{\overline{3} 1} \cdot \tilde{\sigma}\right)^{-1} x_{\overline{3} 2} \cdot \tilde{\sigma}\left(x_{\overline{1} 2} \cdot \tilde{\sigma}\right)^{-1} \\
& \Phi_{1 a}=i\left(x_{\overline{3} 1} \cdot \tilde{\sigma}\right)^{-1} \bar{\theta}_{31 a}-i\left(x_{\overline{2} 1} \cdot \tilde{\sigma}\right)^{-1} \bar{\theta}_{21 a} \\
& \bar{\Phi}_{1}^{a}=i \theta_{12}^{a}\left(x_{\overline{1} 2} \cdot \tilde{\sigma}\right)^{-1}-i \theta_{13}^{a}\left(x_{\overline{1} 3} \cdot \tilde{\sigma}\right)^{-1}
\end{aligned}
$$

Using

$$
x_{\overline{1} 3} \cdot \tilde{\sigma}+x_{\overline{2} 1} \cdot \tilde{\sigma}-4 i \bar{\theta}_{21 a} \theta_{13}^{a}=x_{\overline{2} 3} \cdot \tilde{\sigma}
$$

one can show the consistency condition

$$
Y_{1+} \cdot \sigma=Y_{1-} \cdot \sigma-4 i \Phi_{1 a} \bar{\Phi}_{1}^{a}=-\left(x_{\overline{2} 1} \cdot \sigma\right)^{-1} x_{\overline{2} 3} \cdot \sigma\left(x_{\overline{1} 3} \cdot \sigma\right)^{-1}=\left(Y_{1-} \cdot \sigma\right)^{\dagger}
$$

which is necessary to define $\mathbb{Z}_{1}^{\mathcal{N}}=\left(X^{\mu}, \Theta^{a}, \bar{\Theta}_{a}\right) \in \mathbb{R}^{4 \mid 4 N}$ with $\Theta^{a}, \bar{\Theta}_{a}$ defined according to eq. (5.38).

From eq. (4.90), $\tilde{\mathcal{F}}\left(\widetilde{\mathbb{Z}}_{1}\right)$ transforms infinitesimally as

$$
\begin{aligned}
\delta \tilde{\mathcal{F}}\left(\widetilde{\mathbb{Z}}_{1}\right)= & \left(\begin{array}{cc}
\tilde{\hat{w}}\left(z_{1}\right)-\frac{1}{2} \hat{\lambda}\left(z_{1}\right)+i \frac{1}{2} \hat{\psi}\left(z_{1}\right) & 0 \\
0 & \hat{t}\left(z_{1}\right)+i \frac{2}{N} \hat{\psi}\left(z_{1}\right)
\end{array}\right) \tilde{\mathcal{F}}\left(\widetilde{\mathbb{Z}}_{1}\right) \\
& -\tilde{\mathcal{F}}\left(\widetilde{\mathbb{Z}}_{1}\right)\left(\begin{array}{cc}
\hat{w}\left(z_{1}\right)+\frac{1}{2} \hat{\lambda}\left(z_{1}\right)+i \frac{1}{2} \hat{\psi}\left(z_{1}\right) & 0 \\
0 & \hat{t}\left(z_{1}\right)+i \frac{2}{N} \hat{\psi}\left(z_{1}\right)
\end{array}\right)
\end{aligned}
$$

\section{Superconformal Invariance of Correlation Functions}

In this section we assume that there exist quasi-primary superfields, $\Psi^{I}(z)$ which under the superconformal transformation, $g$, where $z \stackrel{g}{\longrightarrow} z^{\prime}$, transform as

$$
\Psi^{I} \stackrel{g}{\longrightarrow} \Psi^{\prime I} \quad \Psi^{\prime I}\left(z^{\prime}\right)=\Psi^{J}(z) D_{J}^{I}(z ; g)
$$

$D(z ; g)$ obeys the group property so that under the successive superconformal transformations, $g^{\prime \prime}: z \stackrel{g}{\longrightarrow} z^{\prime} \stackrel{g^{\prime}}{\longrightarrow} z^{\prime \prime}$, it satisfies

$$
D(z ; g) D\left(z^{\prime} ; g^{\prime}\right)=D\left(z ; g^{\prime \prime}\right)
$$


and hence also

$$
D(z ; g)^{-1}=D\left(z^{\prime} ; g^{-1}\right)
$$

We choose here $D(z ; g)$ to be a representation of $\mathrm{SO}(1,5) \times \mathrm{Sp}(N) \times \mathrm{D}$, which is a subgroup of the stability group at $z=0$, where $\mathrm{D}$ is the one dimensional group of dilations, and so with $\Psi^{I} \equiv \Psi^{\rho r}$ it may be factorized

$$
D_{J}^{I}(z ; g)=D_{\rho}^{\sigma}(\hat{L}(z ; g)) D_{r}^{s}(U(z ; g)) \Omega(z ; g)^{-\eta}
$$

where $D_{\rho}^{\sigma}(\hat{L}), D_{r}^{s}(U)$ are representations of $\mathrm{G}_{L}, \mathrm{Sp}(N)$ respectively and since $\Omega(z ; g)$ separately satisfies eq.(6.2),$\Omega(z ; g)^{-\eta}$ forms a one dimensional representation of $\mathrm{D}$ with $\eta$, the scale dimension of $\Psi^{\rho r}$.

Infinitesimally

$$
\delta \Psi^{\rho r}(z)=-(\mathcal{L}+\eta \hat{\lambda}(z)) \Psi^{\rho r}(z)-\Psi^{\sigma r}(z) \frac{1}{2}\left(s_{A B}\right)_{\sigma}{ }^{\rho} \hat{\omega}^{A B}(z)-\Psi^{\rho s}(z) \frac{1}{2}\left(t_{i}{ }^{j}\right)_{s}^{r} \hat{T}_{j}{ }^{i}(z)
$$

where $s_{A B}, t_{i}{ }^{j}$ are matrix generators of $\mathrm{SO}(1,5), \mathrm{Sp}(N)$ satisfying

$$
\begin{gathered}
{\left[s_{A B}, s_{C D}\right]=-\eta_{A C} s_{B D}+\eta_{A D} s_{B C}+\eta_{B C} s_{A D}-\eta_{B D} s_{A C}} \\
{\left[t_{i}^{j}, t_{k}^{l}\right]=-\delta_{k}{ }^{j} t_{i}{ }^{l}+\delta_{i}{ }^{l} t_{k}^{j}-\mathcal{E}^{j l} t_{k i}+\overline{\mathcal{E}}_{i k} t^{j l}}
\end{gathered}
$$

where $t_{k i}=t_{i k}=t_{k}{ }^{m} \overline{\mathcal{E}}_{m i}, t^{j l}=t^{l j}=t_{m}{ }^{l} \mathcal{E}^{m j}$. Thus

$$
\left[\frac{1}{2} t_{i}{ }^{j} T_{1 j}{ }^{i}, \frac{1}{2} t_{k}{ }^{l} T_{2 l}{ }^{k}\right]=\frac{1}{2} t_{i}^{j}\left[T_{1}, T_{2}\right]_{j}{ }^{i}
$$

From eqs.4.16, 4.17) using eq.(6.6) we have

$$
\delta_{3} \Psi^{\rho r}=\left[\delta_{2}, \delta_{1}\right] \Psi^{\rho r}
$$

Superconformal invariance for a general $n$-point function requires

$$
\left\langle\Psi_{1}^{\prime I_{1}}\left(z_{1}\right) \Psi_{2}^{\prime I_{2}}\left(z_{2}\right) \cdots \Psi_{n}^{\prime I_{n}}\left(z_{n}\right)\right\rangle=\left\langle\Psi_{1}^{I_{1}}\left(z_{1}\right) \Psi_{2}^{I_{2}}\left(z_{2}\right) \cdots \Psi_{n}^{I_{n}}\left(z_{n}\right)\right\rangle
$$

\subsection{Two-point Correlation Functions}

The solution for the two-point function of the quasi-primary superfields, $\Psi^{\rho r}$, has the general form

$$
\left\langle\Psi^{\rho r}\left(z_{1}\right) \Psi^{\sigma s}\left(z_{2}\right)\right\rangle=C_{\Psi} \frac{I^{\rho \sigma}\left(\hat{\mathcal{X}}_{12}\right) I^{r s}\left(V\left(z_{12}\right)\right)}{\left(\operatorname{det} \mathcal{X}_{12}\right)^{\frac{1}{2} \eta}}
$$


where we define

$$
\hat{\mathcal{X}}_{12}=\frac{\mathcal{X}_{12}}{\left(\operatorname{det} \mathcal{X}_{12}\right)^{\frac{1}{4}}}
$$

and $I^{\rho \sigma}\left(\hat{\mathcal{X}}_{12}\right), I^{r s}\left(V\left(z_{12}\right)\right)$ are tensors transforming covariantly according to the appropriate representations of $\mathrm{G}_{L}, \operatorname{Sp}(N)$ which are formed by decomposition of tensor products of $\hat{\mathcal{X}}_{12}, V\left(z_{12}\right)$, where $V\left(z_{12}\right)$ is given by eq.(4.26).

Under superconformal transformations $I^{\rho \sigma}\left(\hat{\mathcal{X}}_{12}\right), I^{r s}\left(V\left(z_{12}\right)\right)$ satisfy from eqs. (4.58, 4.62)

$$
\begin{gathered}
D\left(\hat{L}\left(z_{1} ; g\right)\right)^{t} I\left(\hat{\mathcal{X}}_{12}\right) D\left(\hat{L}\left(z_{2} ; g\right)\right)=I\left(\hat{\mathcal{X}}_{12}^{\prime}\right) \\
D\left(U\left(z_{1} ; g\right)\right)^{t} I\left(V\left(z_{12}\right)\right) D\left(U\left(z_{2} ; g\right)\right)=I\left(V\left(z_{12}^{\prime}\right)\right)
\end{gathered}
$$

As examples, we consider the spinorial fields, $\phi^{\alpha}(z), \bar{\phi}_{\alpha}(z)$ which transform as

$$
\begin{gathered}
\phi^{\alpha}\left(z^{\prime}\right)=\Omega(z ; g)^{-\eta} \phi^{\beta}(z) \hat{L}_{\beta}^{\alpha}(z ; g) \\
\bar{\phi}_{\alpha}^{\prime}\left(z^{\prime}\right)=\Omega(z ; g)^{-\eta} \hat{L}_{\alpha}^{\beta}\left(z^{\prime} ; g^{-1}\right) \bar{\phi}_{\beta}(z)
\end{gathered}
$$

so that $s_{A B} \rightarrow \frac{1}{2} \gamma_{[A} \tilde{\gamma}_{B]}$.

The two-point functions are

$$
\begin{array}{cc}
\left\langle\phi^{\alpha}\left(z_{1}\right) \phi^{\beta}\left(z_{2}\right)\right\rangle=C_{\phi} \frac{I^{\alpha \beta}\left(\hat{\mathcal{X}}_{12}\right)}{\left(\operatorname{det} \mathcal{X}_{12}\right)^{\frac{1}{2} \eta}} & I^{\alpha \beta}\left(\hat{\mathcal{X}}_{12}\right)=\hat{\mathcal{X}}_{12}^{\alpha \beta} \\
\left\langle\bar{\phi}_{\alpha}\left(z_{1}\right) \bar{\phi}_{\beta}\left(z_{2}\right)\right\rangle=C_{\bar{\phi}} \frac{\bar{I}_{\alpha \beta}\left(\hat{\mathcal{X}}_{12}\right)}{\left(\operatorname{det} \mathcal{X}_{12}\right)^{\frac{1}{2} \eta}} \quad \bar{I}_{\alpha \beta}\left(\hat{\mathcal{X}}_{12}\right)=\left(\hat{\mathcal{X}}_{21}^{-1}\right)_{\alpha \beta}
\end{array}
$$

$I^{\alpha \beta}\left(\hat{\mathcal{X}}_{12}\right), \bar{I}_{\alpha \beta}\left(\hat{\mathcal{X}}_{12}\right)$ satisfy

$$
I^{\alpha \beta}\left(\hat{\mathcal{X}}_{12}\right) \bar{I}_{\gamma \beta}\left(\hat{\mathcal{X}}_{12}\right)=\delta_{\gamma}^{\alpha}
$$

For a vector field, $V^{A}(z)$, where the representation of $\mathrm{G}_{L}$ is given by $\hat{R}_{A}{ }^{B}(z ; g)$, we have

$$
\left\langle V^{A}\left(z_{1}\right) V^{B}\left(z_{2}\right)\right\rangle=C_{V} \frac{I^{A B}\left(\hat{\mathcal{X}}_{12}\right)}{\left(\operatorname{det} \mathcal{X}_{12}\right)^{\frac{1}{2} \eta}} \quad I^{A B}\left(\hat{\mathcal{X}}_{12}\right)=\frac{1}{4} \operatorname{tr}\left(\gamma^{A} \hat{\mathcal{X}}_{12} \gamma^{B} \hat{\mathcal{X}}_{21}\right)
$$

From eqs. 2.11b, A.1a) and using $\hat{\mathcal{X}}_{12}^{t}=\hat{\mathcal{X}}_{21}$, $\operatorname{det} \hat{\mathcal{X}}_{12}=1$ we have

$$
\left(\hat{\mathcal{X}}_{12}^{-1} \tilde{\gamma}_{A} \hat{\mathcal{X}}_{21}^{-1}\right)_{\alpha \beta}=\frac{1}{2} \epsilon_{\alpha \beta \gamma \delta}\left(\hat{\mathcal{X}}_{21} \gamma_{A} \hat{\mathcal{X}}_{12}\right)^{\gamma \delta}
$$


which implies with eq. 2.11a

$$
I_{A B}\left(\hat{\mathcal{X}}_{12}\right)=\frac{1}{4} \operatorname{tr}\left(\gamma_{A} \hat{\mathcal{X}}_{12} \gamma_{B} \hat{\mathcal{X}}_{21}\right)=\frac{1}{4} \operatorname{tr}\left(\tilde{\gamma}_{A} \hat{\mathcal{X}}_{21}^{-1} \tilde{\gamma}_{B} \hat{\mathcal{X}}_{12}^{-1}\right)
$$

Hence $I^{A B}\left(\hat{\mathcal{X}}_{12}\right)$ satisfies

$$
I^{A B}\left(\hat{\mathcal{X}}_{12}\right) I_{C B}\left(\hat{\mathcal{X}}_{12}\right)=\delta_{C}^{A}
$$

Note that $I\left(\hat{\mathcal{X}}_{12}\right) \propto R\left(z_{12} ; i\right)$, where $R(z ; i)$ is given by eq. (3.65).

For a scalar field, $S(z)$ and a symplectic field, $\psi^{i}(z)$, which transforms as

$$
\psi^{\prime i}\left(z^{\prime}\right)=\Omega(z ; g)^{-\eta} \psi^{j}(z) U_{j}{ }^{i}(z ; g)
$$

the two-point functions are

$$
\begin{gathered}
\left\langle S\left(z_{1}\right) S\left(z_{2}\right)\right\rangle=C_{S} \frac{1}{\left(\operatorname{det} \mathcal{X}_{12}\right)^{\frac{1}{2} \eta}} \\
\left\langle\psi^{i}\left(z_{1}\right) \psi^{j}\left(z_{2}\right)\right\rangle=C_{\psi} \frac{V^{i j}\left(z_{12}\right)}{\left(\operatorname{det} \mathcal{X}_{12}\right)^{\frac{1}{2} \eta}} \quad V^{i j}\left(z_{12}\right)=V_{k}^{j}\left(z_{12}\right) \mathcal{E}^{k i}
\end{gathered}
$$

The uniqueness of the two-point function can be shown using the method in 13 .

\subsection{Three-point Correlation Functions}

The solution for the three-point function of the quasi-primary superfields, $\Psi^{\rho r}$, has the general form 13.

$$
\left\langle\Psi_{1}^{\rho r}\left(z_{1}\right) \Psi_{2}^{\sigma s}\left(z_{2}\right) \Psi_{3}^{\tau t}\left(z_{3}\right)\right\rangle=\frac{I^{\sigma \sigma^{\prime}}\left(\hat{\mathcal{X}}_{21}\right) I^{\tau \tau^{\prime}}\left(\hat{\mathcal{X}}_{31}\right) I^{s s^{\prime}}\left(V\left(z_{21}\right)\right) I^{t t^{\prime}}\left(V\left(z_{31}\right)\right) H_{\sigma^{\prime} s^{\prime} \tau^{\prime} t^{\prime}}\left(\widetilde{\mathbb{Z}}_{1}\right)}{\left(\operatorname{det} \mathcal{X}_{31}\right)^{\frac{1}{2} \eta_{3}}\left(\operatorname{det} \mathcal{X}_{12}\right)^{\frac{1}{2} \eta_{2}}}
$$

where $\widetilde{\mathbb{Z}}_{1}^{\tilde{\mathcal{A}}}=\left(\mathrm{Y}_{1}^{A}, \Phi_{1}^{i}\right) \in \mathbb{R}_{-}^{6 \mid 8 N}$ is given by eqs. $\left.4.86,4.89\right)$.

Superconformal invariance (6.9) is equivalent, from eqs.(4.59, 4.92, 6.12a), to

$$
\begin{gathered}
H_{\sigma s \tau t}^{\rho^{\prime} r}(\widetilde{\mathbb{Z}}) D_{\rho^{\prime}}{ }^{\rho}(\hat{L})=D_{\sigma}^{\sigma^{\prime}}(\hat{L}) D_{\tau}^{\tau^{\prime}}(\hat{L}) H_{\sigma^{\prime} s \tau^{\prime} t}^{\rho r}\left(\widetilde{\mathbb{Z}}^{\prime}\right) \\
\widetilde{\mathbb{Z}}^{\prime \tilde{\mathcal{A}}}=\left(Y^{B} \hat{R}_{B}{ }^{A}(\hat{L}), \hat{L}^{-1} \Phi^{i}\right)
\end{gathered}
$$




$$
\begin{gathered}
H_{\sigma s \tau t}^{\rho r^{\prime}}(\widetilde{\mathbb{Z}}) D_{r^{\prime}}{ }^{r}(U)=D_{s}^{s^{\prime}}(U) D_{t}^{t^{\prime}}(U) H_{\sigma s^{\prime} \tau t^{\prime}}^{\rho r}\left(\widetilde{\mathbb{Z}}^{\prime \prime}\right) \\
\widetilde{\mathbb{Z}}^{\prime \prime \tilde{\mathcal{A}}}=\left(Y^{A}, \Phi^{j} U_{j}{ }^{i}\right) \\
H_{\sigma s \tau t}^{\rho r}(\widetilde{\mathbb{Z}})=\lambda^{\eta_{2}+\eta_{3}-\eta_{1}} H^{\rho r}{ }_{\sigma s \tau t}\left(\widetilde{\mathbb{Z}}^{\prime \prime \prime}\right) \\
\widetilde{\mathbb{Z}}^{\prime \prime \prime} \tilde{\mathcal{A}} \\
=\left(\lambda Y^{A}, \lambda^{\frac{1}{2}} \Phi^{i}\right)
\end{gathered}
$$

Note that $\hat{L} \in \mathrm{G}_{L}, U \in \mathrm{Sp}(N), \lambda \in \mathbb{R}$ and $\hat{R}_{B}{ }^{A}(\hat{L})$ is given by eq. (4.65).

In general there may be a finite number, $n$, linearly independent solutions of eq.(6.24a) so that the three-point function depends on $n$ parameters.

\section{Acknowledgements}

I am deeply indebted to Hugh Osborn for his careful guidance throughout the time from the inception of this research to its writing up in this paper.

This work was partly supported by Cambridge Overseas Trust. 


\section{Appendix}

\section{A Notations \& Useful Equations}

Using eqs.(2.7, 2.9) one can derive

$$
\begin{aligned}
& \left(\gamma^{A}\right)_{\alpha \beta}\left(\gamma_{A}\right)_{\gamma \delta}=2 c \epsilon_{\alpha \beta \gamma \delta} \\
& \left(\tilde{\gamma}^{A}\right)^{\alpha \beta}\left(\tilde{\gamma}_{A}\right)^{\gamma \delta}=2 \frac{1}{c} \epsilon^{\alpha \beta \gamma \delta}
\end{aligned}
$$

where $\epsilon_{1234}=\epsilon^{1234}=1$ and $c$ is a constant, equal to the Pfaffian of $\gamma^{0}$, which can be without loss of generality taken to be -1 to get eqs.(2.11a, 2.11b).

Due to the identities 8

$$
\gamma^{[A} \tilde{\gamma}^{B} \gamma^{C]}=-\frac{1}{6} \epsilon_{D E F}^{A B C}{ }^{D} \tilde{\gamma}^{E} \gamma^{F} \quad \tilde{\gamma}^{[A} \gamma^{B} \tilde{\gamma}^{C]}=\frac{1}{6} \epsilon_{D E F}^{A B C} \tilde{\gamma}^{D} \gamma^{E} \tilde{\gamma}^{F}
$$

there are only 10 independent $\gamma^{[A} \tilde{\gamma}^{B} \gamma^{C]}$ and $\tilde{\gamma}^{[A} \gamma^{B} \tilde{\gamma}^{C]}$ separately and both of them form bases of $4 \times 4$ symmetric matrices with the completeness relation

$$
\left(\gamma^{[A} \tilde{\gamma}^{B} \gamma^{C]}\right)_{\alpha \beta}\left(\tilde{\gamma}_{[A} \gamma_{B} \tilde{\gamma}_{C]}\right)^{\gamma \delta}=-24\left(\delta_{\alpha}^{\gamma} \delta_{\beta}^{\delta}+\delta_{\alpha}^{\delta} \delta_{\beta}^{\gamma}\right)
$$

The coefficient on the right hand side may be determined by

$$
\operatorname{tr}\left(\gamma^{[A} \tilde{\gamma}^{B} \gamma^{C]} \tilde{\gamma}_{[D} \gamma_{E} \tilde{\gamma}_{F]}\right)=4 \epsilon_{D E F}^{A B C}-24 \delta_{D}^{[A} \delta_{E}^{B} \delta_{F}^{C]}
$$

A particular convenient choice for $\gamma^{A}, \tilde{\gamma}^{A}$ is

$$
\begin{array}{lll}
\gamma^{\mu}=\left(\begin{array}{cc}
0 & -\sigma^{\mu t} \\
\sigma^{\mu} & 0
\end{array}\right) & \gamma^{4}=\left(\begin{array}{cc}
\bar{\epsilon} & 0 \\
0 & \epsilon
\end{array}\right) & \gamma^{5}=i\left(\begin{array}{cc}
\bar{\epsilon} & 0 \\
0 & -\epsilon
\end{array}\right) \\
\tilde{\gamma}^{\mu}=\left(\begin{array}{cc}
0 & \tilde{\sigma}^{\mu} \\
-\tilde{\sigma}^{\mu t} & 0
\end{array}\right) & \tilde{\gamma}^{4}=-\left(\begin{array}{cc}
\bar{\epsilon}^{-1} & 0 \\
0 & \epsilon^{-1}
\end{array}\right) & \tilde{\gamma}^{5}=i\left(\begin{array}{cc}
\bar{\epsilon}^{-1} & 0 \\
0 & -\epsilon^{-1}
\end{array}\right)
\end{array}
$$

where $\epsilon_{\alpha \beta}, \bar{\epsilon}_{\dot{\alpha} \dot{\beta}}$ are the $2 \times 2$ anti-symmetric matrices, $\epsilon_{12}=\bar{\epsilon}_{12}=1$ with inverses, $\left(\epsilon^{-1}\right)^{\alpha \beta},\left(\bar{\epsilon}^{-1}\right)^{\dot{\alpha} \dot{\beta}}$ and $\left(\sigma^{\mu}\right)_{\alpha \dot{\alpha}},\left(\tilde{\sigma}^{\mu}\right)^{\dot{\alpha} \alpha}, \mu=0,1,2,3$ are $2 \times 2$ matrices satisfying

$$
\begin{array}{ll}
\sigma^{\mu} \tilde{\sigma}^{\nu}+\sigma^{\nu} \tilde{\sigma}^{\mu}=2 \eta^{\mu \nu} & \eta^{\mu \nu}=\operatorname{diag}(+1,-1,-1,-1) \\
\sigma_{\alpha \dot{\alpha}}^{\mu} \tilde{\sigma}_{\mu}^{\dot{\beta} \beta}=2 \delta_{\alpha}{ }^{\beta} \delta_{\dot{\alpha}}^{\dot{\beta}} & \sigma_{\alpha \dot{\alpha}}^{\mu} \sigma_{\mu \beta \dot{\beta}}=2 \epsilon_{\alpha \beta} \bar{\epsilon}_{\dot{\alpha} \dot{\beta}} \\
\epsilon \tilde{\sigma}^{\mu t} \bar{\epsilon}=-\sigma^{\mu} & \bar{\epsilon}^{-1} \sigma^{\mu t} \epsilon^{-1}=-\tilde{\sigma}^{\mu}
\end{array}
$$

\footnotetext{
${ }^{8}$ We put $\epsilon^{012345}=1$ and [] means anti-symmetrizing indices with "strength one".
} 
We define the conjugate of $D_{i \alpha}, \tilde{D}_{i}^{\alpha}$ to be

$$
\bar{D}_{\alpha}^{i}=-\frac{\partial}{\partial \bar{\theta}_{i}^{\alpha}}+i\left(\gamma^{A} \theta^{i}\right)_{\alpha} \frac{\partial}{\partial x^{A}} \quad \overline{\tilde{D}}^{i \alpha}=-\frac{\partial}{\partial \overline{\tilde{\theta}}_{i \alpha}}+i\left(\tilde{\gamma}^{A} \tilde{\theta}^{i}\right)^{\alpha} \frac{\partial}{\partial x^{A}}
$$

so that they satisfy the pseudo-Majorana condition

$$
D_{i \alpha}=\bar{D}_{\alpha}^{j} \overline{\mathcal{E}}_{j i} \quad \tilde{D}_{i}^{\alpha}=\overline{\tilde{D}}^{j \alpha} \overline{\mathcal{E}}_{j i}
$$

\section{B Derivation of Eqs.(3.29, 3.30, 3.31, 3.36, 3.37)}

We recall eqs.(3.20, 3.25)

$$
\begin{gathered}
D_{i \alpha} \tilde{\mathrm{h}}^{\beta \gamma}=\frac{1}{3}\left(\delta_{\alpha}{ }^{\beta} D_{i \delta} \tilde{\mathrm{h}}^{\delta \gamma}-\delta_{\alpha}^{\gamma} D_{i \delta} \tilde{\mathrm{h}}^{\delta \beta}\right) \\
\tilde{\mathrm{h}}(x, \theta)=\tilde{\mathrm{x}}_{-} \mathrm{b}(\theta) \tilde{\mathrm{x}}_{+}+W(\theta) \tilde{\mathrm{x}}_{+}+\tilde{\mathrm{x}}_{-} W^{t}(\theta)+\tilde{\mathrm{A}}(\theta)
\end{gathered}
$$

Writing $\Psi_{\alpha \beta \gamma}=D_{i \alpha} \mathrm{b}_{\beta \gamma}(\theta), \Psi_{\alpha}=\tilde{\mathrm{x}}^{\beta \gamma} \Psi_{\beta \gamma \alpha}$, the $x^{2}$ terms read

$$
3 x^{2} \Psi_{\alpha \beta \gamma}=\mathrm{x}_{\beta \alpha} \Psi_{\gamma}-\mathrm{x}_{\gamma \alpha} \Psi_{\beta}
$$

and so

$$
3 x^{2}\left(\Psi_{\alpha \beta \gamma}+\Psi_{\beta \alpha \gamma}+\Psi_{\gamma \beta \alpha}\right)=2 \mathrm{x}_{\beta \gamma} \Psi_{\alpha}
$$

Contracting with $\tilde{\mathrm{x}}^{\gamma \beta}$ gives

$$
2 \Psi_{\alpha}=3 \tilde{\mathrm{x}}^{\gamma \beta} \Psi_{\alpha \beta \gamma}
$$

which implies $\tilde{\gamma}_{A}^{\beta \gamma}\left(2 D_{i \beta} \mathrm{b}_{\gamma \alpha}+3 D_{i \alpha} \mathrm{b}_{\beta \gamma}\right)=0$, and by eq.(2.9) we get

$$
3 D_{i \alpha} \mathrm{b}_{\beta \gamma}+D_{i \beta} \mathrm{b}_{\gamma \alpha}+D_{i \gamma} \mathrm{b}_{\alpha \beta}=0
$$

which gives $D_{i \alpha} \mathrm{b}_{\beta \gamma}=-\frac{3}{2} D_{i[\alpha} \mathrm{b}_{\beta \gamma]}=0$. Thus, $\mathrm{b}(\theta)$ is independent of $\theta$.

Now the terms linear in $x$ read

$$
3 D_{i \alpha}\left(W(\theta) \tilde{\gamma}^{A}\right)^{[\beta \gamma]}=\delta_{\alpha}{ }^{\beta} D_{i \delta}\left(W(\theta) \tilde{\gamma}^{A}\right)^{[\delta \gamma]}-\delta_{\alpha}^{\gamma} D_{i \delta}\left(W(\theta) \tilde{\gamma}^{A}\right)^{[\delta \beta]}
$$

Contracting with $\gamma_{A \delta \epsilon}$ gives

$$
3 D_{i \alpha} W_{[\delta}^{[\gamma} \delta_{\epsilon]}^{\beta]}=D_{i \eta} W_{[\delta}^{\eta} \delta_{\epsilon]}^{[\beta} \delta_{\alpha}^{\gamma]}+D_{i[\delta} W_{\epsilon]}^{[\beta} \delta_{\alpha}^{\gamma]}
$$


Contracting with $\delta_{\gamma}^{\delta}$ again, we get

$$
2\left(D_{i \alpha} W_{\gamma}^{\beta}-\delta_{\alpha}^{\beta} D_{i \gamma} W_{\delta}^{\delta}\right)=D_{i \delta} W_{\{\alpha}^{\delta} \delta_{\gamma\}}^{\beta}-D_{i\{\alpha} W_{\gamma\}}^{\beta}-3 D_{i\{\alpha} \delta_{\gamma\}}^{\beta} W_{\delta}^{\delta}
$$

The right hand side is symmetric over $\alpha \leftrightarrow \gamma$ and so

$$
D_{i \alpha} W_{\gamma}^{\beta}-\delta_{\alpha}^{\beta} D_{i \gamma} W_{\delta}^{\delta}=D_{i \gamma} W_{\alpha}^{\beta}-\delta_{\gamma}^{\beta} D_{i \alpha} W_{\delta}^{\delta}
$$

Contracting with $\delta_{\beta}^{\alpha}$ gives $D_{i \alpha} W_{\beta}^{\alpha}=4 D_{i \beta} W_{\beta}^{\beta}$. Substituting this back into eq.(B.9) gives

$$
D_{i \alpha} W_{\gamma}^{\beta}-\delta_{\alpha}^{\beta} D_{i \gamma} W_{\delta}^{\delta}=-5\left(D_{i \gamma} W_{\alpha}^{\beta}-\delta_{\gamma}^{\beta} D_{i \alpha} W_{\delta}^{\delta}\right)
$$

which implies eq. (3.30) and

$$
D_{j \delta} D_{i \alpha} W_{\gamma}^{\beta}=-\delta_{\alpha}^{\beta} D_{i \gamma} D_{j \delta} W_{\epsilon}^{\epsilon}=-D_{i \gamma} D_{j \alpha} W_{\delta}^{\beta}=\delta_{\gamma}^{\beta} D_{j \alpha} D_{i \delta} W_{\epsilon}^{\epsilon}
$$

Contracting with $\delta_{\beta}^{\gamma}$ gives

$$
D_{j \beta} D_{i \alpha} W_{\gamma}^{\gamma}=4 D_{j \alpha} D_{i \beta} W_{\gamma}^{\gamma}
$$

which implies $D_{j \beta} D_{i \alpha} W_{\gamma}^{\gamma}=0$ and so eq.(3.31).

Acting $D_{j \delta}$ on eq. (3.35) reads

$$
\frac{9}{2} D_{j \delta} D_{i \alpha} \tilde{\mathrm{A}}^{\beta \gamma}=D_{i \delta} D_{j \epsilon} \tilde{\mathrm{A}}^{\epsilon[\beta} \delta_{\alpha}^{\gamma]}+\delta_{\delta}^{[\beta} \delta_{\alpha}^{\gamma]} D_{i \epsilon} D_{j \eta} \tilde{\mathrm{A}}^{\epsilon \eta}
$$

Contracting with $\delta_{\beta}^{\alpha}$ gives

$$
3 D_{j \delta} D_{i \alpha} \tilde{\mathrm{A}}^{\alpha \gamma}+D_{i \delta} D_{j \alpha} \tilde{\mathrm{A}}^{\alpha \gamma}=-\delta_{\delta}^{\gamma} D_{i \epsilon} D_{j \eta} \tilde{\mathrm{A}}^{\epsilon \eta}
$$

The right hand side of which is symmetric over $i \leftrightarrow j$, and so

$$
D_{i \alpha} D_{j \beta} \tilde{\mathrm{A}}^{\beta \gamma}=D_{j \alpha} D_{i \beta} \tilde{\mathrm{A}}^{\beta \gamma}=-\frac{1}{4} \delta_{\alpha}^{\gamma} D_{i \epsilon} D_{j \eta} \tilde{\mathrm{A}}^{\epsilon \eta}
$$

Substituting this back into eq.(B.14) reads eq.(3.36). Eq.(3.36) implies

$$
D_{i \alpha} D_{j \beta} D_{k \gamma} \tilde{\mathrm{A}}^{\beta \gamma}=D_{j \beta} D_{k \gamma} D_{i \alpha} \tilde{\mathrm{A}}^{\beta \gamma}=-\frac{1}{4} D_{j \alpha} D_{k \beta} D_{i \gamma} \tilde{\mathrm{A}}^{\beta \gamma}
$$

and so $D_{i \alpha} D_{j \beta} D_{k \gamma} \tilde{\mathrm{A}}^{\beta \gamma}=0$. Therefore, we get eq.(3.37). 


\section{C $(N, 0)$ Superconformal Algebra}

We write the superconformal generators in general as

$$
\chi=a^{A} P_{A}+\bar{\varepsilon}_{i} Q^{i}+\lambda D+\frac{1}{2} \omega^{A B} M_{A B}+b^{A} K_{A}+\bar{\rho}_{i} S^{i}+\frac{1}{2} T_{i}{ }^{j} A_{j}{ }^{i}
$$

where the $\operatorname{Sp}(N)$ generators, $A_{i}{ }^{j}$, satisfy $A^{\dagger}=-A, A^{t} \mathcal{E}+\mathcal{E} A=0$.

The $(N, 0)$ superconformal algebra can now be obtained by imposing

$$
\left[\chi_{1}, \chi_{2}\right]=-i \chi_{3}
$$

where $\chi_{i}$ is defined by substituting $\left(a^{A}, \varepsilon^{i}, \lambda, \omega^{A B}, b^{A}, \rho^{i}, T_{i}{ }^{j}\right)$ by the corresponding coefficient appearing in eq.(3.78). From this expression, we can read off the following $(N, 0)$ superconformal algebra.

- Poincaré algebra

$$
\begin{aligned}
& {\left[P_{A}, P_{B}\right]=0 \quad\left[M_{A B}, P_{C}\right]=i\left(\eta_{A C} P_{B}-\eta_{B C} P_{A}\right)} \\
& {\left[M_{A B}, M_{C D}\right]=i\left(\eta_{A C} M_{B D}-\eta_{A D} M_{B C}-\eta_{B C} M_{A D}+\eta_{B D} M_{A C}\right)}
\end{aligned}
$$

- Supersymmetry algebra

$$
\begin{gathered}
{\left[P_{A}, Q^{i}\right]=0 \quad\left\{Q^{i}, Q^{j}\right\}=2 \mathcal{E}^{i j} \gamma^{A} P_{A}} \\
{\left[M_{A B}, Q^{i}\right]=i \frac{1}{2} \gamma_{[A} \tilde{\gamma}_{B]} Q^{i}}
\end{gathered}
$$

- Special superconformal algebra

$$
\begin{array}{cc}
{\left[K_{A}, K_{B}\right]=0} & {\left[M_{A B}, K_{C}\right]=i\left(\eta_{A C} K_{B}-\eta_{B C} K_{A}\right)} \\
{\left[K_{A}, S^{i}\right]=0} & \left\{S^{i}, S^{j}\right\}=2 \mathcal{E}^{i j} \tilde{\gamma}^{A} K_{A} \\
& {\left[M_{A B}, S^{i}\right]=i \frac{1}{2} \tilde{\gamma}_{[A} \gamma_{B]} S^{i}}
\end{array}
$$

- Cross terms between $(P, Q)$ and $(K, S)$

$$
\begin{gathered}
{\left[P_{A}, K_{B}\right]=2 i\left(M_{A B}+\eta_{A B} D\right)} \\
{\left[P_{A}, S^{i}\right]=\tilde{\gamma}_{A} Q^{i} \quad\left[K_{A}, Q^{i}\right]=\gamma_{A} S^{i}} \\
\left\{Q_{\alpha}^{i}, S^{j \beta}\right\}=i \mathcal{E}^{i j}\left(2 \delta_{\alpha}^{\beta} D+\left(\gamma^{[A} \tilde{\gamma}^{B]}\right)_{\alpha}^{\beta} M_{A B}\right)-4 i \delta_{\alpha}^{\beta} A^{i j}
\end{gathered}
$$


- Dilations

$$
\begin{array}{cc}
{\left[D, P_{A}\right]=-i P_{A}} & {\left[D, K_{A}\right]=i K_{A}} \\
{\left[D, Q^{i}\right]=-i \frac{1}{2} Q^{i}} & {\left[D, S^{i}\right]=i \frac{1}{2} S^{i}} \\
{[D, D]=\left[D, M_{A B}\right]=\left[D, A_{i}{ }^{j}\right]} & =0
\end{array}
$$

- R-symmetry, $\operatorname{Sp}(N)$

$$
\begin{gathered}
{\left[A_{i}{ }^{j}, A_{k}{ }^{l}\right]=i\left(\delta_{k}{ }^{j} A_{i}{ }^{l}-\delta_{i}{ }^{l} A_{k}{ }^{j}+\mathcal{E}^{j l} A_{k i}-\overline{\mathcal{E}}_{i k} A^{j l}\right)} \\
{\left[A_{i}{ }^{j}, Q^{k}\right]=i\left(\mathcal{E}^{j k} \bar{Q}_{i}^{t}-\delta_{i}{ }^{k} Q^{j}\right)} \\
{\left[A_{i}{ }^{j}, S^{k}\right]=i\left(\mathcal{E}^{j k} \bar{S}_{i}^{t}-\delta_{i}{ }^{k} S^{j}\right)} \\
{\left[A_{i}{ }^{j}, P_{A}\right]=\left[A_{i}{ }^{j}, K_{A}\right]=\left[A_{i}{ }^{j}, M_{A B}\right]=0}
\end{gathered}
$$

where $A_{i j}=A_{j i}=A_{i}{ }^{k} \overline{\mathcal{E}}_{k j}, A^{i j}=A^{j i}=A_{k}{ }^{j} \mathcal{E}^{k i}$.

\section{Realization of $\mathrm{O}(2,6)$ structure in $M$}

We exhibit explicitly the relation of the six-dimensional conformal group to $\mathrm{O}(2,6)$ by introducing eight-dimensional gamma matrices with $R=0,1, \cdots, 7$

$$
\left(\begin{array}{cc}
0 & \Sigma^{R} \\
\tilde{\Sigma}^{R} & 0
\end{array}\right)
$$

$\Sigma^{R}, \tilde{\Sigma}^{R}$ satisfy

$$
\Sigma^{R} \tilde{\Sigma}^{S}+\Sigma^{S} \tilde{\Sigma}^{R}=2 G^{R S}
$$

where $G^{R S}=\operatorname{diag}(+1,-1,-1, \cdots,-1,+1)$. In particular, here we choose $\Sigma^{R}, \tilde{\Sigma}^{R}$ as

$$
\begin{array}{lll}
\Sigma^{A}=\left(\begin{array}{cc}
\tilde{\gamma}^{A} & 0 \\
0 & \gamma^{A}
\end{array}\right) & \Sigma^{6}=\left(\begin{array}{cc}
0 & i \\
-i & 0
\end{array}\right) & \Sigma^{7}=\left(\begin{array}{cc}
0 & i \\
i & 0
\end{array}\right) \\
\tilde{\Sigma}^{A}=\left(\begin{array}{cc}
\gamma^{A} & 0 \\
0 & \tilde{\gamma}^{A}
\end{array}\right) & \tilde{\Sigma}^{6}=-\Sigma^{6} & \tilde{\Sigma}^{7}=-\Sigma^{7}
\end{array}
$$


$\Sigma_{R}, \tilde{\Sigma}_{R}$ satisfy

$$
\begin{aligned}
\left(\begin{array}{cc}
0 & \tilde{\gamma}^{0} \\
\gamma^{0} & 0
\end{array}\right) \Sigma_{R}\left(\begin{array}{cc}
0 & \gamma^{0} \\
\tilde{\gamma}^{0} & 0
\end{array}\right) & =\tilde{\Sigma}_{R}^{\dagger}=\Sigma^{R} \\
\left(\begin{array}{ll}
0 & 1 \\
1 & 0
\end{array}\right) \Sigma_{R}\left(\begin{array}{ll}
0 & 1 \\
1 & 0
\end{array}\right) & =-\tilde{\Sigma}_{R}^{t}
\end{aligned}
$$

For the matrix, $M$, given in eq.(3.79), we may now express the $8 \times 8$ part in terms of $\Sigma^{[R} \tilde{\Sigma}^{S]}$

$$
\left(\begin{array}{cc}
\omega+\frac{1}{2} \lambda & -i \tilde{\mathrm{a}} \\
-i \mathrm{~b} & \tilde{\omega}-\frac{1}{2} \lambda
\end{array}\right)=\frac{1}{4} \omega_{R S} \Sigma^{[R} \tilde{\Sigma}^{S]}
$$

where $\omega_{67}, \omega_{A 6}, \omega_{A 7}$ are given by

$$
\omega_{67}=\lambda \quad \omega_{A 6}=a_{A}-b_{A} \quad \omega_{A 7}=a_{A}+b_{A}
$$

$\Sigma^{R S} \equiv \frac{1}{2} \Sigma^{\left[R \tilde{\Sigma}^{S]}\right.}$ generates the Lie algebra of $\mathrm{O}(2,6)$

$$
\left[\Sigma^{R S}, \Sigma^{T U}\right]=-G^{R T} \Sigma^{S U}+G^{R U} \Sigma^{S T}+G^{S T} \Sigma^{R U}-G^{S U} \Sigma^{R T}
$$

Eqs.(3.82, 3.83), the conditions on $M$, are satisfied by eq.(D.4).

The result on superinversion (3.67) corresponds to the reflection of the sixth axis.

\section{E Superconformally Covariant Operators}

In general acting on a quasi-primary superfield, $\Psi^{\rho r}(z)$, with the spinor derivative, $D_{i \alpha}$, does not lead to a quasi-primary field. For a superfield, $\Psi^{\rho r}$, from eqs. (4.10, 4.11) we have

$$
\begin{aligned}
D_{i \alpha} \delta \Psi^{\rho r}= & -\left(\mathcal{L}+\left(\eta+\frac{1}{2}\right) \hat{\lambda}\right) D_{i \alpha} \Psi^{\rho r}+\tilde{\hat{\omega}}_{\alpha}{ }^{\beta} D_{i \beta} \Psi^{\rho r}-D_{i \alpha} \Psi^{\sigma r} \frac{1}{2}\left(s_{A B} \hat{\omega}^{A B}\right)_{\sigma}{ }^{\rho} \\
& +\hat{T}_{i}{ }^{j} D_{j \alpha} \Psi^{\rho r}-D_{i \alpha} \Psi^{\rho s} \frac{1}{2}\left(t_{j}{ }^{k} \hat{T}_{k}{ }^{j}\right)_{s}^{r}+2 \overline{\hat{\rho}}_{j \beta}\left(\Psi Y_{i \alpha}^{j \beta}\right)^{\rho r}
\end{aligned}
$$

We may connect the generator of $\mathrm{G}_{L}$ to $\mathrm{SO}(1,5)$ by

$$
s_{\alpha}{ }^{\beta} \equiv-\frac{1}{2} s_{A B}\left(\gamma^{[A} \tilde{\gamma}^{B]}\right)_{\alpha}{ }^{\beta}
$$

where

$$
\left[s_{\alpha}{ }^{\beta}, s_{\gamma}{ }^{\delta}\right]=2 \delta_{\alpha}^{\delta} s_{\gamma}{ }^{\beta}-2 \delta_{\gamma}{ }^{\beta} s_{\alpha}{ }^{\delta}
$$


so that $s_{\alpha}{ }^{\beta} \tilde{\hat{\omega}}_{\beta}^{\alpha}=s_{A B} \hat{\omega}^{A B}$ and then

$$
Y_{i \alpha}^{j \beta}=\eta \delta_{i}^{j} \delta_{\alpha}^{\beta}-\delta_{i}{ }^{j} s_{\alpha}{ }^{\beta}+2 t_{i}^{j} \delta_{\alpha}^{\beta}
$$

To ensure that $D_{i \alpha} \Psi^{\rho r}$ is quasi-primary it is necessary that the terms proportional to $\hat{\rho}$ vanish and this can be achieved by restricting $D_{i \alpha} \Psi^{\rho r}$ to an irreducible representation of $\mathrm{G}_{L}, \mathrm{Sp}(N)$ and choosing a particular value of $\eta$ so that $\Psi Y=0$. The change of the scale dimension, $\eta \rightarrow \eta+\frac{1}{2}$, in eq.(E.1) is also apparent from eq.(4.67)

$$
D_{i \alpha}=\Omega(z ; g)^{1 / 2} \hat{L}_{\alpha}^{\beta}(z ; g) U_{i}^{j}(z ; g) D_{j \beta}^{\prime}
$$

As an illustration we consider tensorial fields, $\Psi_{\beta_{1} \cdots \beta_{m} i_{1} \cdots i_{n}}^{\alpha_{1} \cdots \alpha_{l}}$, which transform as

$$
\begin{aligned}
\delta \Psi_{\beta_{1} \cdots \beta_{m} i_{1} \cdots i_{n}}^{\alpha_{1} \cdots \alpha_{l}}= & -(\mathcal{L}+\eta \hat{\lambda}) \Psi_{\beta_{1} \cdots \beta_{m} i_{1} \cdots i_{n}}^{\alpha_{1} \cdots \alpha_{l}} \\
& -\sum_{p=1}^{l} \Psi_{\beta_{1} \cdots \beta_{m} i_{1} \cdots i_{n}}^{\alpha_{1} \cdots \gamma \alpha_{l}} \tilde{\hat{\omega}}_{\gamma}{ }^{\alpha_{p}}+\sum_{q=1}^{m} \tilde{\hat{\omega}}_{\beta_{q}}{ }^{\gamma} \Psi_{\beta_{1} \cdots \gamma \beta_{m} i_{1} \cdots i_{n}}^{\alpha_{1} \cdots \alpha_{l}} \\
& +\sum_{r=1}^{n} \hat{T}_{i_{r}}{ }^{j} \Psi_{\beta_{1} \cdots \beta_{m} i_{1} \cdots j \cdots i_{n}}^{\alpha_{1} \cdots \alpha_{l}}
\end{aligned}
$$

In this case we have

$$
\begin{aligned}
\left(\Psi Y_{i \alpha}^{j \beta}\right)_{\beta_{1} \cdots \beta_{m} i_{1} \cdots i_{n}}^{\alpha_{1} \cdots \alpha_{l}}= & 2 \delta_{i}{ }^{j}\left(-\sum_{p=1}^{l} \delta_{\alpha}^{\alpha_{p}} \Psi_{\beta_{1} \cdots \beta_{m} i_{1} \cdots i_{n}}^{\alpha_{1} \cdots \beta \cdots \alpha_{l}}+\sum_{q=1}^{m} \delta_{\beta_{q}}^{\beta} \Psi_{\beta_{1} \cdots \alpha_{l} \cdots \beta_{m} i_{1} \cdots i_{n}}^{\alpha_{1} \cdots \alpha_{2}}\right) \\
& +2 \delta_{\alpha}{ }^{\beta}\left(\sum_{r=1}^{n}\left(\overline{\mathcal{E}}_{i i_{r}} \mathcal{E}^{j k} \Psi_{\beta_{1} \cdots \beta_{m} i_{1} \cdots k i_{n}}^{\alpha_{1} \cdots \alpha_{l}}-\delta_{i_{r}}{ }^{j} \Psi_{\beta_{1} \cdots \beta_{m} i_{1} \cdots i i_{n}}^{\alpha_{1} \cdots \alpha_{l}}\right)\right) \\
& +\left(\eta+\frac{1}{2} l-\frac{1}{2} m\right) \delta_{i}{ }^{j} \delta_{\alpha}{ }^{\beta} \Psi_{\beta_{1} \cdots \beta_{m} i_{1} \cdots i_{n}}^{\alpha_{1} \cdots \alpha_{l}}
\end{aligned}
$$

In particular, eq.(E.7) shows that the following are quasi-primary

$$
\begin{array}{ll}
D_{(i(\alpha} \Psi_{\left.\left.\beta_{1} \cdots \beta_{m}\right) i_{1} \cdots i_{n}\right)} & \text { if } \quad \eta=2 n-\frac{3}{2} m \\
D_{(i[\alpha} \Psi_{\left.\left.\beta_{1} \cdots \beta_{m}\right] i_{1} \cdots i_{n}\right)} & \text { if } \quad \eta=2 n+\frac{5}{2} m \\
D_{(i|\alpha|} \Psi_{\left.i_{1} \cdots i_{n}\right)}^{\left[\alpha \alpha_{1} \cdots \alpha_{l}\right]} & \text { if } \quad \eta=\frac{15}{2}-\frac{5}{2} l+2 n
\end{array}
$$




$$
\begin{array}{ll}
D_{(i|\alpha|} \Psi_{\left.i_{1} \cdots i_{n}\right)}^{\left(\alpha \alpha_{1} \cdots \alpha_{l}\right)} & \text { if } \quad \eta=\frac{15}{2}+\frac{3}{2} l+2 n \\
D_{(i|\alpha|} \Psi_{\left.i_{1} \cdots i_{n}\right)}^{\beta}-\frac{1}{4} \delta_{\alpha}^{\beta} D_{(i|\gamma|} \Psi_{\left.i_{1} \cdots i_{n}\right)}^{\gamma} & \text { if } \quad \eta=2 n-\frac{1}{2}
\end{array}
$$

where ( ), [] denote the usual symmetrization, anti-symmetrization respectively and obviously eqs.(E.8b, E.8c) are nontrivial if $m, l \leq 3$.

Now we consider the case where more than one spinor derivative, $D_{i \alpha}$, act on a quasiprimary superfield. In this case, acting on a general tensor field, there are inhomogeneous terms proportional to $\overline{\hat{\rho}}_{j \beta}$, as in eq.(E.1), but also

$$
D_{i \alpha} \overline{\hat{\rho}}_{j \beta}=\frac{1}{2} i \mathrm{~b}_{\alpha \beta} \overline{\mathcal{E}}_{i j}
$$

The latter terms can be eliminated by symmetrizing all the symplectic indices, $i, j, \cdots$, while the $\overline{\hat{\rho}}$ terms may vanish by a suitable choice of the scale dimension, $\eta$.

Hence, the following are quasi-primary

$$
\begin{array}{ll}
D_{\left(i _ { 1 } \left[\alpha_{1}\right.\right.} \cdots D_{i_{k} \alpha_{k}} \Psi_{\left.\left.\beta_{1} \cdots \beta_{m}\right] j_{1} \cdots j_{n}\right)} & \text { if } \quad \eta=\frac{5}{2} m+2 n+2 k-2 \\
D_{\left(i_{1}\left|\alpha_{1}\right|\right.} \cdots D_{i_{k}\left|\alpha_{k}\right|} \Psi_{\left.j_{1} \cdots j_{n}\right)}^{\left[\alpha_{1} \cdots \alpha_{k} \beta_{1} \cdots \beta_{l}\right]} & \text { if } \quad \eta=8+2 n-\frac{5}{2} l-\frac{1}{2} k
\end{array}
$$

Note that

$$
D_{(i(\alpha} D_{j) \beta)}=0
$$

\section{References}

[1] B. Nilsson. Superspace Action for a 6-Dimensional Non-extended Supersymmetric Yang-Mills Theory. Nucl. Phys., B174: 335, 1980.

[2] P. Howe, G. Sierra and P. Townsend. Supersymmetry in Six Dimensions. Nucl. Phys., B221: 331, 1983.

[3] J. Koller. A Six-dimensional Superspace Approach to Extended Superfields. Nucl. Phys., B222: 319, 1983.

[4] P. Howe, K. Stelle and P. West. $N=1, d=6$ Harmonic Superspace. Class. Quantum Grav., 2: 815, 1985. 
[5] P. Howe and A. Umerski. Anomaly Multiplets in Six and Ten Dimensions. Phys. Lett., 198B: 57, 1987.

[6] N. Seiberg. Non-trivial Fixed Points of The Renormalization Group in Six Dimensions. Phys. Lett., B390: 169, 1997.

[7] K. Intriligator. RG Fixed Points in Six Dimensions via Branes at Orbifold Singularities. Nucl. Phys., B496: 177, 1997.

[8] G. Dall'Agata, K. Lechner and M. Tonin. Covariant actions for $N=1, D=6$ Supergravity theories with chiral bosons. Nucl. Phys., B512: 179, 1998.

[9] P. Claus, R. Kallosh and A. Van Proeyen. M 5-brane and Superconformal $(0,2)$ tensor multiplet in 6 dimensions. Nucl. Phys., B518: 117, 1998.

[10] W. Nahm. Supersymmetries and Their Representations. Nucl. Phys., B135: 149, 1978.

[11] V. Kac. A Sketch of Lie Superalgebra Theory. Comm. Math. Phys., 53: 31, 1977.

[12] N. Seiberg. Notes on Theories with 16 Supercharges. Proceedings of The Trieste Spring School 1997. hep-th/9705117.

[13] J-H Park. N=1 Superconformal Symmetry in Four Dimensions. Int. J. Mod. Phys. A, 13: 1743, 1998. hep-th/9703191.

[14] H. Osborn and A. Petkou. Implications of Conformal Invariance in Field Theories for General Dimensions. Ann. Phys., 231: 311-361, 1994.

[15] J. Erdmenger and H. Osborn. Conserved Currents and the Energy-Momentum Tensor in Conformally invariant Theories for General Dimensions. Nucl. Phys., B483: 431, 1997.

[16] I. L. Buchbinder and S. M. Kuzenko. Ideas and Methods of Supersymmetry and Supergravity or a Walk through Superspace. Institute of Physics Publishing Ltd., 1995.

[17] P. West. Introduction to Rigid Supersymmetric Theories. hep-th/9805055.

[18] T. Kugo and P. Townsend. Supersymmetry and the Division Algebras. Nucl. Phys., B221: 357, 1983.

[19] C. Grojean and J. Mourad. Superconformal 6D $(2,0)$ theories in Superspace. hepth/9807055. 